\title{
Probing leptoquarks and heavy neutrinos at the LHeC
}

\author{
Sanjoy Mandal ${ }^{*}$ and Nita Sinha ${ }^{\dagger}$ \\ The Institute of Mathematical Sciences, C.I.T Campus, Taramani, Chennai 600 113, India \\ and Homi Bhabha National Institute, BARC Training School Complex, \\ Anushakti Nagar, Mumbai 400085, India \\ Manimala Mitra \\ Institute of Physics, Sachivalaya Marg, Bhubaneswar 751005, India \\ and Homi Bhabha National Institute, BARC Training School Complex, \\ Anushakti Nagar, Mumbai 400085, India
}

(Received 22 July 2018; published 5 November 2018; corrected 31 January 2019)

\begin{abstract}
We explore leptoquark production and decay for the $\tilde{R}_{2}$ class of models at the proposed $e^{-} p$ collider $\mathrm{LHeC}$, planned to operate with $150 \mathrm{GeV}$ electron and $7 \mathrm{TeV}$ proton beams. In addition to the coupling of the leptoquark with the lepton and jet, the model also has right-handed neutrinos coupled to the leptoquark. We analyze the collider signatures of a number of final states that can originate from leptoquark decay into the standard model particles, as well as the final states that originate from further decay of the heavy neutrinos produced from the leptoquark. We find that the final state $\ell^{-}+n-$ jets $(1 \leq n \leq 2)$ has the largest discovery prospect, more than $5 \sigma$ with only a few $\mathrm{fb}^{-1}$ of data to probe a leptoquark of mass $1.1 \mathrm{TeV}$, even with a generic set of cuts. The significance falls sharply with increasing leptoquark mass. However, with $100 \mathrm{fb}^{-1}$ of data, a $5 \sigma$ discovery for leptoquarks of mass up to $1.4 \mathrm{TeV}$ is still achievable. Also, for the same luminosity final state $\bar{b} \ell^{+} \tau^{-}+n-$ jets $(n \geq 2)+\mathbb{E}_{T}$, resulting from the cascade decay of the leptoquark to a $\bar{t}$ and right-handed neutrino, followed by further decays of $\bar{t}$ and the neutrino, is expected to yield a rather large number of events $(\approx 180)$.
\end{abstract}

DOI: 10.1103/PhysRevD.98.095004

\section{INTRODUCTION}

Discovery of physics beyond the Standard Model (SM) continues to be the aim of most high energy physicists today. In spite of the so far unsuccessful direct searches for new particles or clear indications for the existence of any kind of new physics at the Large Hadron Collider (LHC), issues of the existence of dark matter, baryon asymmetry of the Universe, nonzero neutrino masses, etc., are compelling enough for one to believe that there must exist physics beyond the SM. A number of beyond Standard Model extensions have been proposed in the literature. The minimal supersymmetric standard model, large extra dimensions, seesaw models of neutrino mass generation, leptoquarks, and grand unified theories, are a few among them.

\footnotetext{
*smandal@imsc.res.in nita@imsc.res.in

*manimala@iopb.res.in
}

Published by the American Physical Society under the terms of the Creative Commons Attribution 4.0 International license. Further distribution of this work must maintain attribution to the author(s) and the published article's title, journal citation, and DOI. Funded by SCOAP ${ }^{3}$.
Leptoquarks (LQs) are hypothetical particles, which make leptons couple directly to quarks and vice versa [1-3]. In the Pati-Salam model, they emerged from the unification of quarks and leptons [4]. They also exist in grand unification theories based on $\mathrm{SU}(5)$ [5] and $\mathrm{SO}(10)$ [6-15]. They are also expected to exist at the TeV scale in extended technicolor models [16-19]. LQs can be either of scalar or vector nature. Using the SM representation of quarks and leptons, all possible LQ states can be classified, with six scalar and six vector LQ multiplets under the SM gauge group [20]. Among the different classes, the scalar LQ $\tilde{R}_{2}$ is interesting, as it is one of the multiplets that allows for matter stability [21]. Moreover, it also couples to righthanded neutrinos (RH neutrinos). The $\mathrm{RH}$ neutrinos can generate light neutrino masses through the seesaw mechanism [22-35]. In seesaw, light neutrino masses are generated through the $d=5$ lepton number violating operator [28]. The high scale UV completed models include the gauge singlet RH neutrino (type-I, inverse seesaw), $S U(2)_{L}$ triplet scalar, and fermion (type-II and type-III).

In the case of type-I seesaw [22-25], the light neutrinos acquire masses through mixings with additional Majorana $\mathrm{RH}$ neutrinos. To account for the tiny neutrino masses, the mass scale of these Majorana neutrinos has to be very close 
to the gauge coupling unification scale, in which case these massive RH neutrinos will remain inaccessible at LHC and at other near future colliders. For the present and near future colliders to be able to probe the RH neutrinos their masses have to be within the experimental reach, and the mixing with the active neutrinos, referred to as active-sterile mixing, has to be sizable. TeV scale $\mathrm{RH}$ neutrinos with substantially large active-sterile mixings are, however, possible to accommodate in type-I seesaw if cancellation exists in the light neutrino mass matrix [36]. The inverse seesaw mechanism [33-35] is another such scenario, where the TeV scale or even smaller RH neutrino masses with sizable active-sterile mixing can exist. In this scheme, in addition to the SM particles there are gauge singlet neutrinos with opposite lepton numbers $(+1$ and -1$)$. The light neutrino mass matrix is given in terms of the Dirac neutrino mass term $m_{D} \sim Y_{\nu} v$ (with $v$ being the electroweak vacuum expectation value and $Y_{\nu}$ a generic Yukawa coupling), the heavy neutrino mass scale $M_{R}$ and, a small lepton number violating $(\Delta L=2)$ mass term $\mu$, which ensures that the $M_{R}$ scale remains close to $\mathrm{TeV}$ or less, with order one Yukawa coupling. The light neutrino mass matrix in this case is $m_{\nu} \sim\left(m_{D}^{2} / M_{R}^{2}\right) \mu$. While the heavy neutrino states may lie within the kinematic reach of the LHC, their production cross section falls rapidly with increasing masses and smaller active-sterile neutrino mixing. Large active-sterile mixing is possible to obtain in other seesaw secenarios as well, such as linear seesaw $[37,38]$ and extended seesaw [39-43]. A substantial rise in the production cross section of the RH neutrinos is feasible in the presence of LQs. This has been explored recently for the LHC in Ref. [44] for inverse seesaw, where a number of final states have been analyzed in detail. Leptoquark models have also been tested recently for fitting the IceCube events [45]. For the heavy neutrino searches at $\mathrm{LHeC}$ in the inverse seesaw model, see Ref. [46] and for the LNV signal at LHeC, see Ref. [47]. Similar studies for heavy neutrino searches also have been carried out in Refs. [48,49].

In this work, we consider a particular type of scalar leptoquark $\tilde{R}_{2}$, which transforms as $\tilde{R}_{2} \in\left(3,2, \frac{1}{6}\right)$ under the SM gauge group $S U(3)_{c} \times S U(2)_{L} \times U(1)_{Y}$, and for the $\mathrm{RH}$ neutrino, we adopt a model independent framework. $\tilde{R}_{2}$ is a genuine LQ with fermion number $F=3 B+L=0$. The color charge of the LQ will enable their copious production at the LHC. Moreover, at $e^{-} p$ colliders like $\mathrm{LHeC}$, they can be resonantly produced. The $\mathrm{LHeC}$ is a proposed $e^{-} p$ collider in the $\mathrm{TeV}$ regime after HERA, supposed to be built in the LHC tunnel [50]. LHeC will use a newly built electron beam of $60 \mathrm{GeV}$, up to possibly $150 \mathrm{GeV}$, to collide with the intense $7 \mathrm{TeV}$ proton beam of the LHC. LHeC is expected to operate with $100 \mathrm{fb}^{-1}$ integrated luminosity, and is complementary to the $p p$ collider LHC [51]. The RH neutrino, being coupled to the LQ, can be produced from LQ decay. The decay of LQ into a lepton and a jet, and the decay of the RH neutrino in different SM states give rise to a plethora of model signatures, that we study in detail. We show that among all the final states, $\ell^{-}+n-$ jets $(1 \leq n \leq 2)$ has the highest LQ discovery prospect, even with generic sets of cuts. Additionally, we also carry out an in-depth analysis for a few other channels that arise due to the decay of a heavy neutrino. We show that with judicious application of selection cuts the channels $\ell^{-}+n-\operatorname{jets}(n \geq 3)$, and $\ell^{+} \tau^{-} \bar{b}+\mathbb{E}_{T}+n-$ jets $(n \geq 2)$ can be made background free.

The discussion of the paper goes as follows: in Sec. II, and Sec. III, we review the model and the theory constraints. Following that, in Sec. IV, we discuss the production and decay of LQs at LHeC. In the subsequent sections, Sec. V, Sec. VI, and Sec. VII, we present a detailed collider analysis and discuss the discovery prospects of different final states. Finally, in Sec. VIII, we summarize.

\section{MODEL}

We consider the scalar LQ $\tilde{R}_{2}$ charged as $(3,2,1 / 6)$ under the SM gauge group. In the presence of the $\mathrm{RH}$ neutrinos $N_{R}$ the LQ has additional interaction $[2,3,20]$,

$$
\mathcal{L}=-Y_{i j} \bar{d}_{R}^{i} \tilde{R}_{2}^{a} \epsilon^{a b} L_{L}^{j, b}+Z_{i j} \bar{Q}_{L}^{i, a} \tilde{R}_{2}^{a} N_{R}^{j}+\text { H.c. }
$$

where $i, j=1,2,3$ are flavor indices and $a, b=1,2$ are $S U(2)_{L}$ indices. We assume that there are three right-chiral neutrinos $N_{R}^{j}(j=1,2,3), Y_{i j}$, and $Z_{i j}$ are the elements of arbitrary complex $3 \times 3$ Yukawa coupling matrices. Note that $\tilde{R}_{2}$ comprises two LQs. One has $Q=\frac{2}{3}$, and the other has $Q=-\frac{1}{3}$. Upon expansion, the Lagrangian becomes

$$
\begin{aligned}
\mathcal{L}= & -Y_{i j} \bar{d}_{R}^{i} e_{L}^{j} \tilde{R}_{2}^{2 / 3}+\left(Y U_{\mathrm{PMNS}}\right)_{i j} \bar{d}_{R}^{i} \nu_{L}^{j} \tilde{R}_{2}^{-1 / 3} \\
& +\left(V_{\mathrm{CKM}} Z\right)_{i j} \bar{u}_{L}^{i} N_{R}^{j} \tilde{R}_{2}^{2 / 3}+Z_{i j} \bar{d}_{L}^{i} N_{R}^{j} \tilde{R}_{2}^{-1 / 3}+\text { H.c. },
\end{aligned}
$$

where the superscript of the LQ fields denote the electric charge of a given $S U(2)_{L}$ doublet component of $\tilde{R}_{2} ; U_{\mathrm{PMNS}}$ and $V_{\mathrm{CKM}}$ are the Pontecorvo-Maki-NakagawaSakata (PMNS) and Cabibbo-Kobayashi-Maskawa (CKM) matrices. At the $e^{-} p$ machine $\tilde{R}_{2}^{\frac{1}{3}}$ cannot be resonantly produced. Hence, the expected cross section for the production of $\tilde{R}_{2}^{\frac{1}{3}}$ is small. Therefore, in this work, we consider only $\tilde{R}_{2}^{\frac{2}{3}}$ and study its production and decay.

The charged current and neutral current interactions of the RH neutrinos are parametrized in a model-independent way as follows,

$$
-\mathcal{L}_{\mathrm{CC}}=\frac{g}{\sqrt{2}} W_{\mu}^{-} \bar{\ell} \gamma^{\mu} P_{L} V_{\ell j} N_{j}+\text { H.c. }
$$

and

$$
-\mathcal{L}_{\mathrm{NC}}=\frac{g}{2 \cos \theta_{w}} Z_{\mu}\left\{\left(U_{\mathrm{PMNS}}^{\dagger} V\right)_{i j} \bar{\nu}_{i} \gamma^{\mu} P_{L} N_{j}+\text { H.c. }\right\} .
$$


The interaction of the heavy neutrinos with Higgs boson has the following form:

$$
-\mathcal{L}_{H}=\frac{g M_{j}}{4 M_{W}} H\left\{\left(U_{\mathrm{PMNS}}^{\dagger} V\right)_{i j} \bar{\nu}_{i} P_{R} N_{j}+\text { H.c. }\right\}
$$

In the above, $P_{L / R}=\left(1 \mp \gamma^{5}\right) / 2$ is the left and rightchirality projection operator, and $V$ is the mixing matrix through which light neutrinos mix with the RH neutrinos. We consider a diagonal basis for the charged leptons.

For the RH neutrino, coupled with LQ, we do not assume any particular model. Instead, we are interested in different frameworks of RH neutrinos that can lead to large activesterile mixing, so that the heavy neutrinos decay inside the detector. It is widely known that a number of different frameworks can generate large active-sterile mixing, including inverse and linear seesaw [33-35,37,38], extended seesaw [39-43], and the cancellation framework [36]. In the inverse seesaw, light SM neutrino masses are extremely tiny, owing to the small lepton number violating parameter of the model. The active-sterile neutrino mixing is not constrained from light neutrino masses in this model. Active-sterile mixing up to $\mathcal{O}\left(10^{-2}\right)$ is allowed from experimental data [52-63]. In extended seesaw, or double seesaw [64], the RH neutrino gets mass due to seesaw, and light neutrino masses are generated due to twofold seesaw. In other frameworks, such as, cancellation, small light neutrino masses are generated due to cancellation between different RH neutrino contributions in the mass matrix [36]. The active-sterile mixing is yet unconstrained from neutrino data. In all the above mentioned frameworks, owing to the charged current and neutral current interactions as well as the interaction with the Higgs, specified above in Eqs. (3), (4), and (5), the RH neutrino $N$ can decay to a number of SM particles, including $l^{ \pm} W^{\mp}, \nu Z$, and $\nu H$. The branching ratio of these three decays is $\mathrm{Br}(\mathrm{N} \rightarrow \mathrm{lW}): \mathrm{Br}(\mathrm{N} \rightarrow \nu \mathrm{Z}): \mathrm{Br}(\mathrm{N} \rightarrow \nu \mathrm{H}) \simeq 0.6: 0.3: 0.1$, once RH neutrino mass becomes larger than the Higgs mass $M_{N}>M_{H}$ and $M_{N}<200 \mathrm{GeV}[57,65]$.

In the following sections, we first consider the resonant production of a LQ and its decay to a lepton and jet. We next consider the production of heavy neutrinos from LQ decay, and discuss the discovery prospect of the LQ in a number of channels. As mentioned before, we consider the prompt decays of heavy neutrino for the analysis of the RH neutrino signature, that occurs due to large active-sterile neutrino mixing. We compare between the usual charged current (CC) production of heavy neutrinos vs the alternate production from LQ decay. We show that the production from LQ decay dominates over the CC production mode by an order of magnitude for active-sterile mixing $V \lesssim 10^{-2}$.

\section{CONSTRAINTS ON LEPTOQUARK COUPLINGS}

The couplings of the LQs to fermions are constrained by low energy precision observables such as atomic parity violation, Kaon decays, etc. We assume the Yukawa coupling matrix elements $Y_{i j}=\delta_{i j} Y_{i i}$ and $Z_{i j}=\delta_{i j} Z_{i i}$, where, $i, j=1,2,3$. Hence the LQ couples exclusively to a lepton and a quark of the same generation, although it can have nonzero couplings to fermions of more than one generation.

(i) LQs have been searched for and studied in the context of $e^{+} e^{-}$[66-70], ep [71-76], $p \bar{p}$ [77-81], and $p p$ [82-87] colliders. The present tightest bounds are from the LHC [88-92]. The LHC has studied the process $p p \rightarrow \mathrm{LQ} \overline{\mathrm{LQ}} \rightarrow \ell j \ell j$ for LQs of first, second, and third generations. Nonobservation of any new physics at the LHC has ruled out LQs of masses up to $1.1 \mathrm{TeV}$ at $95 \%$ C.L. for the LQ decaying to $e j$ with $100 \%$ branching ratio [89]. For the second generation, the bound is even more stringent $M_{\mathrm{LQ}}>1.5 \mathrm{TeV}$ at $95 \%$ C.L. [92]. For the third generation, the bound is $M_{\mathrm{LQ}}>$ $900 \mathrm{GeV}$ at $95 \%$ C.L. [91]. At the LHC, numerous QCD diagrams contribute to the LQ pair production. For illustration, we show only one representative gluon initiated diagram in the left panel of Fig. 1. However, with nonzero Yukawa couplings, significantly large contribution to the LQ pair production may arise through a single $t$ channel diagram (see the right panel of Fig. 1). The pair production cross section at the LHC can be parametrized as [93]

$$
\begin{aligned}
\sigma_{\text {pair }}\left(Y_{i i}, M_{\mathrm{LQ}}\right)= & a_{0}\left(M_{\mathrm{LQ}}\right)+a_{2}\left(M_{\mathrm{LQ}}\right)\left|Y_{i i}\right|^{2} \\
& +a_{4}\left(M_{\mathrm{LQ}}\right)\left|Y_{i i}\right|^{4},
\end{aligned}
$$

where the three terms correspond to the QCD pair production, an interference term, and $t$-channel production. In Fig. 2, we show the variation of the LQ pair-production cross section with the Yukawa coupling $Y_{11}$.

For small Yukawa coupling $Y_{11}$, LQ pair production is mostly governed by QCD, as can be seen from the straight line up to $Y_{11} \sim 0.5$ in Fig. 2. For intermediate Yukawa couplings there exists a region with negative interference between QCD diagrams and the $t$-channel diagram, where the total cross section decreases [93], resulting in a mild dip in the cross section for coupling beyond 0.5, which is seen in Fig. 2. For large Yukawa coupling $Y_{i i}$, the $t$-channel process dominates and significantly enhances the cross section. The right panel of Fig. 2 shows the limit on the firstgeneration scalar LQ pair production times the branching fraction to the $e j$ final state as a function of the mass. For the branching fraction LQ $\rightarrow e j$ as $100 \%$, the bound on the pair production of LQ becomes $\sigma(p p \rightarrow$ LQ LQ $) \lesssim 3 \mathrm{fb}$, for LQ mass $1.1 \mathrm{TeV}$. Comparing the left and right 

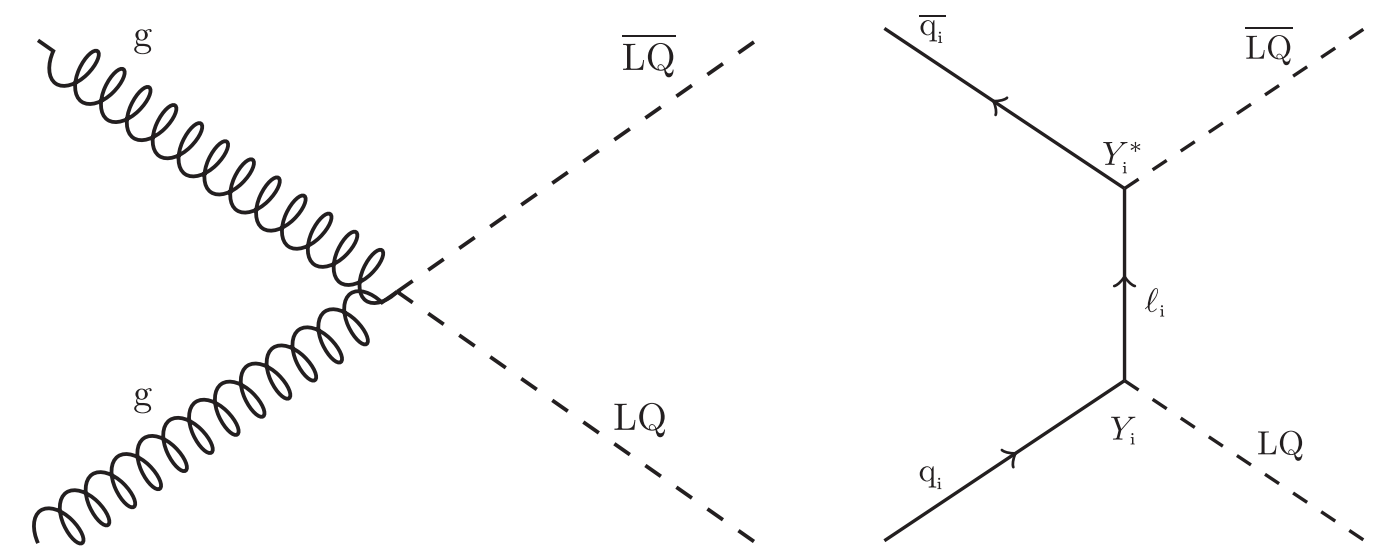

FIG. 1. Left panel: Feynman diagram for the gluon-initiated LQ pair-production process at the LHC. Right panel: the same, but for the quark-initiated processes.
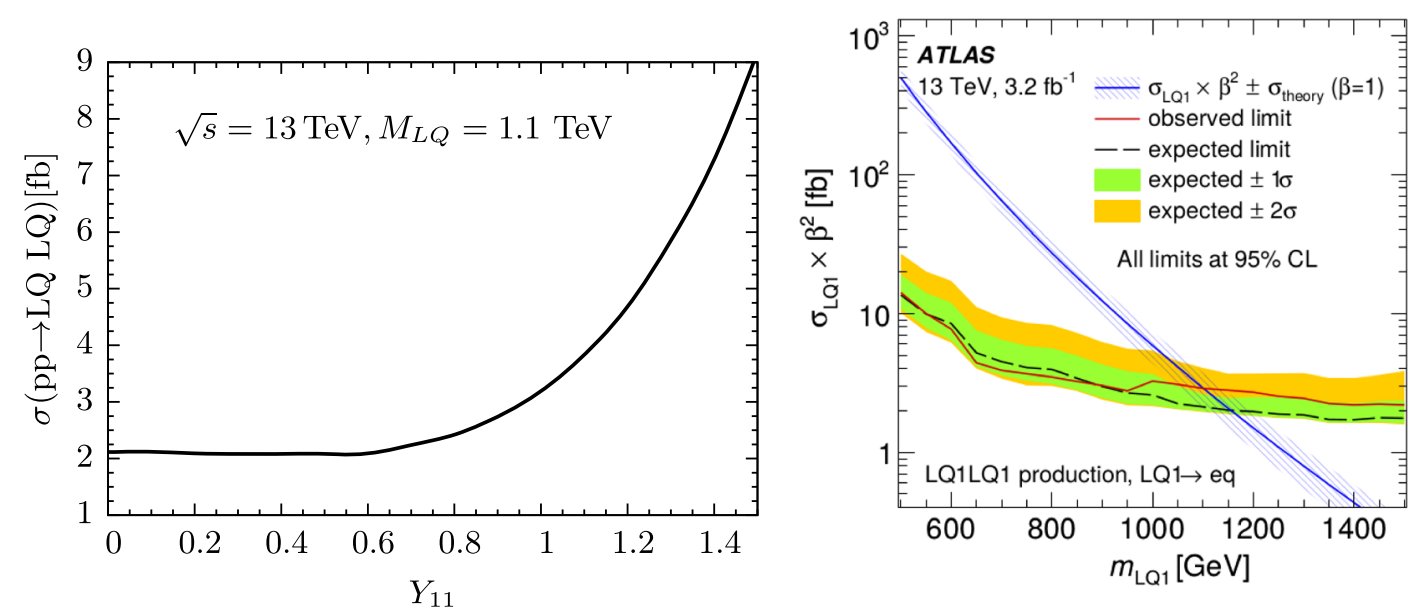

FIG. 2. Left panel: variation of the production cross section with Yukawa coupling for $\sqrt{s}=13$ TeV. Right panel: limit on the scalar LQ pair-production time branching fraction to $e q$ final state as a function of mass for first-generation LQs. The yellow and green bands represent the $2 \sigma$ and $1 \sigma$ expected limits. The NLO prediction is shown in the blue curve with uncertainty due to the choice of the PDF set and the renormalization or factorization scale [89].

panel of Fig. 2, one can see that the limit on the cross section for a LQ of mass $1.1 \mathrm{TeV}$ will be inconsistent with a Yukawa coupling larger than 1 .

It is obvious from Eq. (1) that for the LQ to have $100 \%$ branching ratio in the $\mathrm{LQ} \rightarrow e j$ decay mode, the coupling $Z$ needs to be zero. Allowing a nonzero value for coupling $Z$ will open up new decay modes, such as $\bar{t} N$ for LQs, and hence will lower the stringent bound on LQs. We, however, adopt a conservative approach, and in order to be consistent with the LHC results for the first generation of LQs, throughout our study, we consider $M_{\mathrm{LQ}} \geq 1.1 \mathrm{TeV}$. Additionally, we also keep both the couplings nonzero.

(ii) The present bound on coupling $Y$ from atomic parity violation are $Y_{d e}<0.34\left(\frac{M_{\mathrm{LQ}}}{1 \mathrm{TeV}}\right), Y_{u e}<0.36\left(\frac{M_{\mathrm{LQ}}}{1 \mathrm{TeV}}\right)$ [93]. These bounds are extracted under the assumption that only one of the two contributions is present at a given moment. These bounds allow large coupling for a larger mass of LQs, and place a stringent constraint for lighter LQs.

(iii) The most stringent bound on the diagonal couplings of $\tilde{R}_{2}{ }^{\frac{2}{3}}$ comes from the LFV decay mode $K_{L} \rightarrow \mu^{-} e^{+}$, as this is a tree-level process. Following Refs. [93,94], the bound is given by $\left|Y_{s \mu} Y_{d e}^{*}\right|<$ $2.1 \times 10^{-5}\left(\frac{M_{\mathrm{LQ}}}{1 \mathrm{TeV}}\right)^{2}$. In order to satisfy both the APV and LFV constraints, for $Y_{d e} \sim \mathcal{O}(0.1)$, the other coupling $Y_{s \mu}$ has to be tiny. We consider $Y_{s \mu}$ to be zero and a large value $(0.3)$ for $Y_{d e}$ to get a large production cross section of LQs at LHeC.

We discuss the production of LQs and its decay to different final states in the next section, for the benchmark points, which are in agreement with the described constraints. 


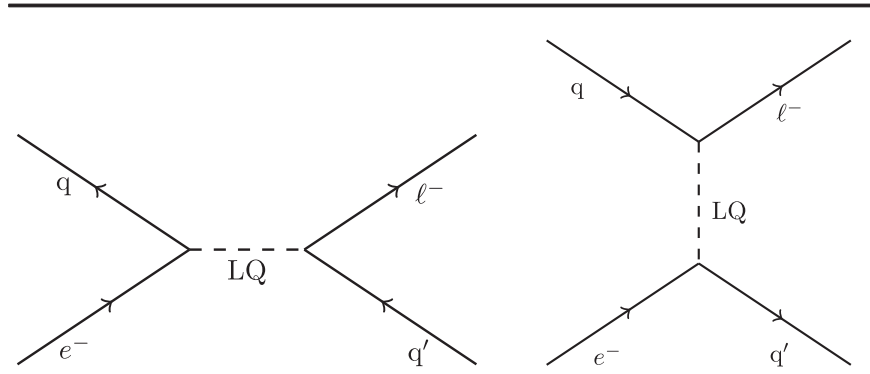

FIG. 3. Feynman diagram for $e^{-} p \rightarrow \ell j$.

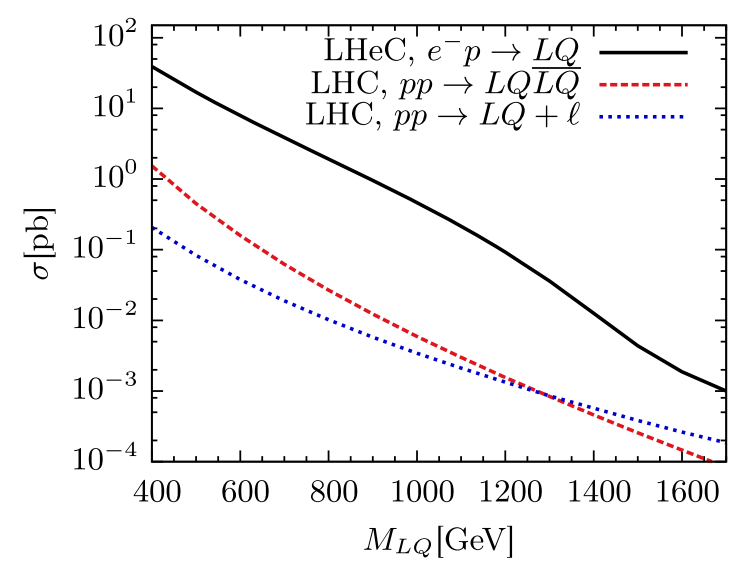

FIG. 4. Comparison of the cross section for LQ production at the LHC and the LHeC. The c.m. energy for the LHC is $\sqrt{s}=13 \mathrm{TeV}$. For the $\mathrm{LHeC}$, we use an electron beam of $150 \mathrm{GeV}$ and proton beam of $7 \mathrm{TeV}$, respectively. The coupling $Y_{d e}$ has been varied as $0.3 \frac{M_{\mathrm{LQ}}}{1 \mathrm{TeV}}$, in agreement with the APV constraints.

\section{LEPTOQUARK PRODUCTION AND ITS DECAYS}

At $e^{-} p$ colliders, scalar LQs can be resonantly produced through the $s$-channel process as shown in the left panel of Fig. 3, and decay to a lepton and a jet. In addition, LQs can also be a $t$-channel mediator for the process $e^{-} p \rightarrow l^{-} j$, that we consider in our analysis (shown in the right panel of Fig. 3).

The production cross section of a $\mathrm{LQ}$ at $\mathrm{LHeC}$, as well as that for both the single and pair production at LHC, are shown in Fig. 4 for varying LQ mass. Clearly, the LHeC cross section is more than both the pair production, as well as the single production of LQs associated with a charged lepton at LHC. The higher LQ production cross section as well as the lower background at $\mathrm{LHeC}$ will allow more precise studies for probing LQs and RH neutrinos. Once produced, the LQ can decay into a number of final states, including (a) a quark-lepton pair that gives rise to a single charged lepton and a light jet, (b) a light jet and a heavy neutrino, and (c) a top quark accompanied with a heavy neutrino. These heavy neutrinos appearing from the decays of the LQ can again be more easily probed at $\mathrm{LHeC}$ through its decay products. Note that, for all these processes, the LQ can also mediate as a $t$-channel mediator. For (b) and (c), there is also a $t$-channel contribution from a $W$ gauge boson mediator, but significantly smaller for the active-sterile mixing $V \lesssim 10^{-2}$. We give numerical estimates in Sec. VI. However, during computation (in Fig. 5 and for the collider analysis), we consider all the contributions together.

For our computations, the LQ mass has been set to 1.1 TeV. We choose three benchmark points, with the three heavy neutrino masses and the LQ couplings $Y_{i i}$ and $Z_{i i}$ chosen such that they are consistent with all the constraints mentioned in Sec. III, as well as with the neutrino oscillation data [46]. These parameters for the benchmark points have been specified in Table I. The production cross section for these three processes at $\mathrm{LHeC}$, with electron and proton beam energies of $150 \mathrm{GeV}$ and $7 \mathrm{TeV}$, respectively, are also shown in Fig. 5 and in Fig. 6 as a function of the couplings and LQ mass respectively.

The general expression for the two body decay of a scalar LQ to $\ell_{i} q$ and $N_{i} q$ final states is given by

$$
\begin{aligned}
& \Gamma\left(\mathrm{LQ} \rightarrow \ell_{i} q\right) \\
& \quad=\frac{\left|Y_{i i}\right|^{2}}{16 \pi M_{\mathrm{LQ}}^{3}} \lambda^{\frac{1}{2}}\left(M_{\mathrm{LQ}}^{2}, m_{\ell_{i}}^{2}, m_{q^{2}}\right)\left(M_{\mathrm{LQ}}^{2}-m_{\ell_{i}}^{2}-m_{q}^{2}\right), \\
& \Gamma\left(\mathrm{LQ} \rightarrow N_{i} q\right) \\
& \quad=\frac{\left|Z_{i i}\right|^{2}}{16 \pi M_{\mathrm{LQ}}^{3}} \lambda^{\frac{1}{2}}\left(M_{\mathrm{LQ}}^{2}, M_{N_{i}}^{2}, m_{q^{2}}\right)\left(M_{\mathrm{LQ}}^{2}-M_{N_{i}}^{2}-m_{q}^{2}\right) .
\end{aligned}
$$

In the massless limit of leptons and quarks, the branching ratios are given by

$$
\begin{aligned}
& \beta\left(\mathrm{LQ} \rightarrow \ell_{i} q\right)=\frac{\left|Y_{i i}\right|^{2}}{\sum_{i}\left(\left|Y_{i i}\right|^{2}+\left|Z_{i i}\right|^{2}\right)} \quad \text { and } \\
& \beta\left(\mathrm{LQ} \rightarrow N_{i} q\right)=\frac{\left|Z_{i i}\right|^{2}}{\sum_{i}\left(\left|Y_{i i}\right|^{2}+\left|Z_{i i}\right|^{2}\right)} .
\end{aligned}
$$

TABLE I. Benchmark parameters and production cross section for $\ell j, j N_{1}$, and $\bar{t} N_{3}$ at the LHeC with electron and proton beam energy $150 \mathrm{GeV}$ and $7 \mathrm{TeV}$, respectively. The LQ mass is considered as $1.1 \mathrm{TeV}$.

\begin{tabular}{lccccr}
\hline \hline Benchmarks & $M_{N_{1,2,3}}$ & $Y$ & $Z$ & Process & $\sigma(\mathrm{fb})$ \\
\hline BP1 & $(150,1000,1000)$ & $(0.3,0,0)$ & $(0,0,0)$ & $\ell j$ & 221 \\
BP2 & $(150,1000,1000)$ & $(0.3,0,0)$ & $(1,0,0)$ & $j N_{1}$ & 242 \\
BP3 & $(1000,1000,150)$ & $(0.3,0,0)$ & $(0,0,1)$ & $\bar{t} N_{3}$ & 222 \\
\hline \hline
\end{tabular}



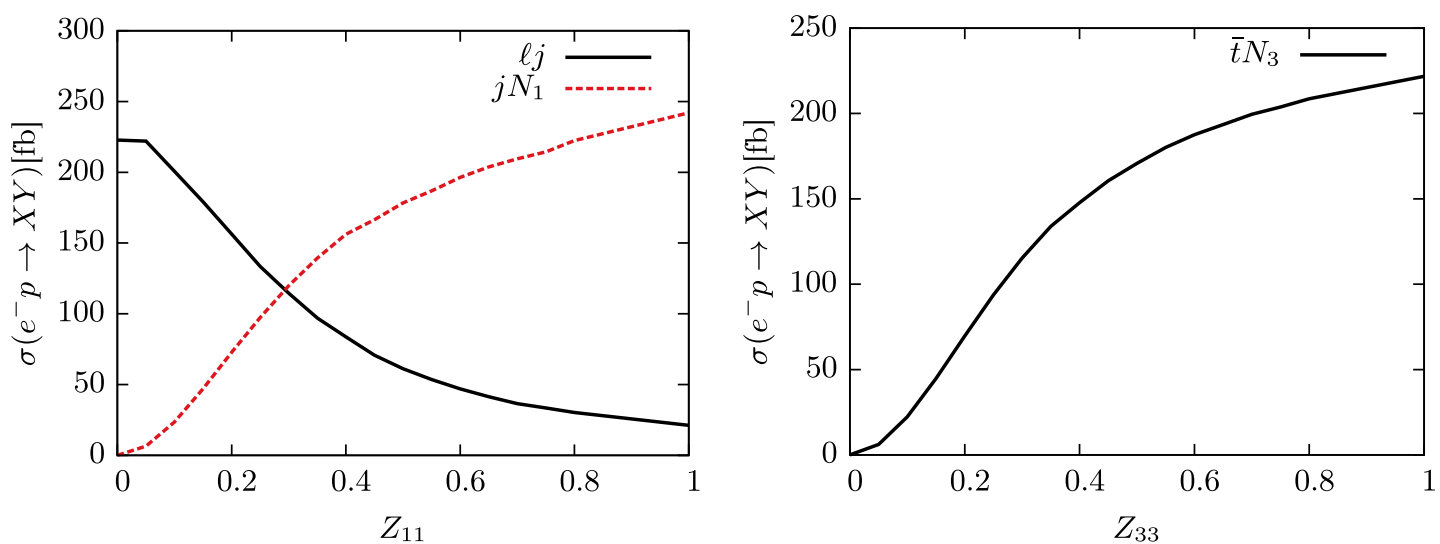

FIG. 5. Production cross section for $\ell j, j N_{1}$, and $\bar{t} N_{3}$ at the LHeC with varying coupling $Z_{11}$. We have considered a $150 \mathrm{GeV}$ electron beam colliding with a $7 \mathrm{TeV}$ proton beam. The LQ mass has been set to $1.1 \mathrm{TeV}$. For $l^{-} j, j N_{1}$, the coupling $Y_{11}=0.3, Z_{11}$ is varying and for $\bar{t} N_{3}$ production, the coupling $Y_{11}=0.3, Z_{33}$ is varying; the rest of the Yukawa couplings have been set to zero.

At an $e^{-} p$ collider LQs can be resonantly produced, followed by their decay. Hence, we can write the cross section approximately as

$$
\begin{aligned}
& \sigma\left(e^{-} p \rightarrow \ell_{i} q \text { or } N_{i} q\right) \\
& \quad \approx \sigma\left(e^{-} p \rightarrow \mathrm{LQ}\right) \cdot \beta\left(\mathrm{LQ} \rightarrow \ell_{i} q \text { or } N_{i} q\right) .
\end{aligned}
$$

As can be seen from Eq. (9), with increasing coupling $Z_{11}$ the branching ratio of $\sigma\left(e^{-} p \rightarrow j N_{1}\right)$ increases, while $\sigma\left(e^{-} p \rightarrow l j\right)$ decreases. This results in a larger cross section for $\sigma\left(e^{-} p \rightarrow j N_{1}\right)$ for larger $Z_{11}$. Cross section for the other channel $e^{-} p \rightarrow \bar{t} N_{3}$ is also large for a large value of $Z_{33}$. The values of the cross section in $\mathrm{fb}$, for three benchmark points are given in the last column of Table I. As can be seen, the production cross section at $\mathrm{LHeC}$ is fairly large, approximately $\sigma \sim 221-242 \mathrm{fb}$ for the chosen benchmark points. As we will show in the next section, folded with branching ratios of heavy neutrino, top quark,

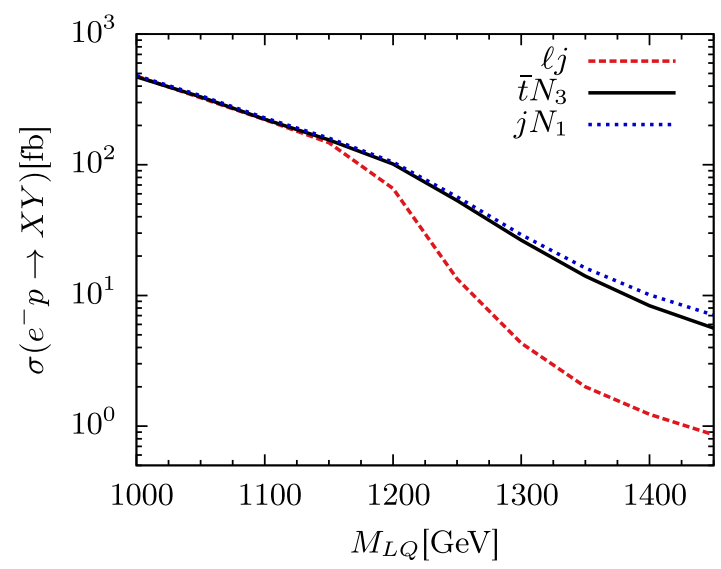

FIG. 6. Production cross section for $\ell j, j N_{1}$, and $t N_{3}$ with varying LQ mass $M_{\mathrm{LQ}}$. For $l^{-} j, j N_{1}$, and $\bar{t} N_{3}$ production we adopt $\mathrm{BP} 1, \mathrm{BP} 2$, and $\mathrm{BP}$, respectively. the total cross section for the different final states will be sizable.

\section{COLLIDER ANALYSIS}

We implemented the model in FeynRules [95] and generated the model files for MadGraph5_aMC@NLO (v2_5_5) [96] to calculate the parton level cross section for signals and background. For the collider simulation part, we passed the MadGraph generated parton level events to PYTHIA (v6.4.28) [97], where subsequent decay, initial state radiation, final state radiation, and hadronization have been carried out. The jets are reconstructed by an anti- $\kappa_{t}$ algorithim [98] implemented in the Fastjetpackage [99] with radius parameter $R=0.4$. For the analysis of signal and background events we use the following set of basic cuts:

(1) Electrons and muons in the final state should have the following transverse momentum and pseudorapidity $p_{T}^{\ell}>20 \mathrm{GeV},\left|\eta^{\ell}\right|<2.5$.

(2) Jets are ordered in $p_{T}$, jets should have $p_{T}^{j}>$ $40 \mathrm{GeV}$ and $\left|\eta^{j}\right|<2.5$.

(3) Photons are counted if $p_{T}^{\gamma}>10 \mathrm{GeV}$ and $\left|\eta^{\gamma}\right|<2.5$.

(4) Jets should be separated by $\Delta R_{j j}>0.5$.

(5) Leptons should be separated by $\Delta R_{\ell \ell}>0.2$.

(6) Leptons and photons isolation $\Delta R_{\ell \gamma}>0.2$.

(7) Jets and leptons should be separated by $\Delta R_{\ell j}>0.4$.

(8) Hadronic activity within a cone of radius 0.3 around a lepton must be limited to $\sum p_{T}^{\text {hadron }}<0.2 p_{T}^{\ell}$, where $p_{T}^{\ell}$ is the transverse momentum of lepton within the specified cone.

Because of the initial and final state radiations, additional jets will be present in the final states considered. For the inverse seesaw framework, lepton number violation (LNV) is dictated by the parameter $\mu_{X}$, which is negligibly small. Therefore, the cross section for LNV dilepton final states will be suppressed. For the framework where light neutrino masses are generated as a result of cancellation, sizable lepton number violation can, however, be present. Below, 
TABLE II. Signal and background cross sections for the final state $\ell^{-}+n-$ jets $(1 \leq n \leq 2)$ after different cuts. BP1 has been used for this final state.

\begin{tabular}{lccc}
\hline \hline Cuts & Final states & Signal $(\mathrm{fb})$ & Background (fb) \\
\hline No cuts & $\ell^{-}+n-$ jets $(1 \leq n \leq 2)$ & 220 & $2.96 \times 10^{6}$ \\
Basic cuts & $\ell^{-}+n-$ jets $(1 \leq n \leq 2)$ & 159 & $4.08 \times 10^{5}$ \\
Leading lepton $p_{T}$ cut & $+p_{T}^{\ell^{-}}(>400 \mathrm{GeV})$ & 118 & 178 \\
LQ invariant mass cut & $+\mid M_{\mathrm{LQ}}-M_{\ell_{1} j_{1}} \leq 100 \mathrm{GeV}$ & 101 & 0 \\
\hline \hline
\end{tabular}

we adopt a conservative approach, and only consider lepton number conserving signatures. A number of signatures, including single lepton and multijet, dilepton associated with multijet and missing energy, and multilepton associated with missing energy and $b$ jet have been analyzed in the subsequent sections.

\section{SIGNALS AND BACKGROUND}

\section{A. Signal I: $\ell^{-}+n-\operatorname{jets}(1 \leq n \leq 2)$}

The single-lepton associated with jet is the easiest channel to probe LQs. LQs, once produced resonantly, can directly decay to a charged lepton and a jet. Additionally, the $t$-channel contribution, as shown in Fig. 3 will also be
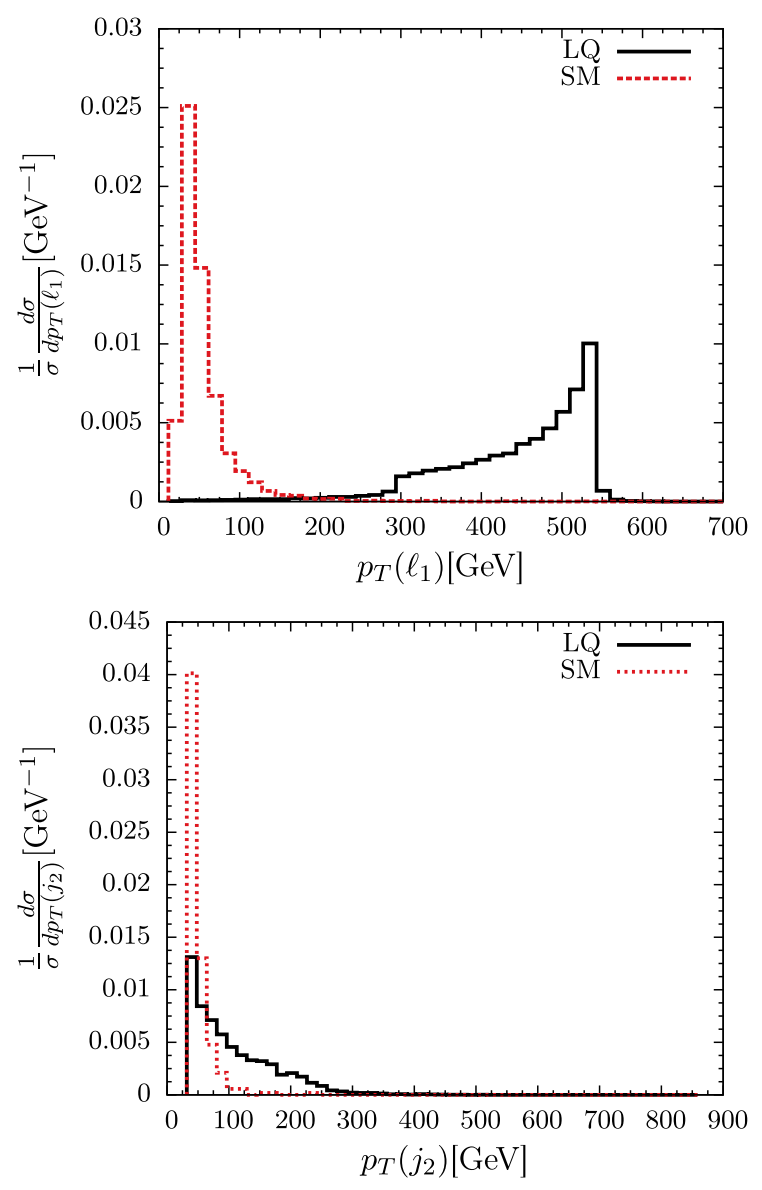

present. Therefore, the parton level final state is $\ell^{-}+n-$ jets $(n=1)$. Additional jets will be present due to ISR, FSR. We demand the final state should contain $\ell^{-}$and number of jets $1 \leq n-$ jets $\leq 2$. The main backgrounds arise from the SM process, such as, $e^{-} p \rightarrow \ell^{-} j, \ell^{-} j j$, which are significantly larger as compared to the signal. From Table II, the signal cross section is $220 \mathrm{fb}$, while the background cross section is $3 \times 10^{6} \mathrm{fb}$. We use a number of cuts on different kinematic variables to reduce the background.

In Fig. 7 we have shown the transverse momentum of the leading lepton, leading and subleading jet, as well as the invariant mass distribution of the leading jet and leading lepton, both for the signal and background. Evidently, for a very heavy LQ, a high- $p_{T}$ cut on the
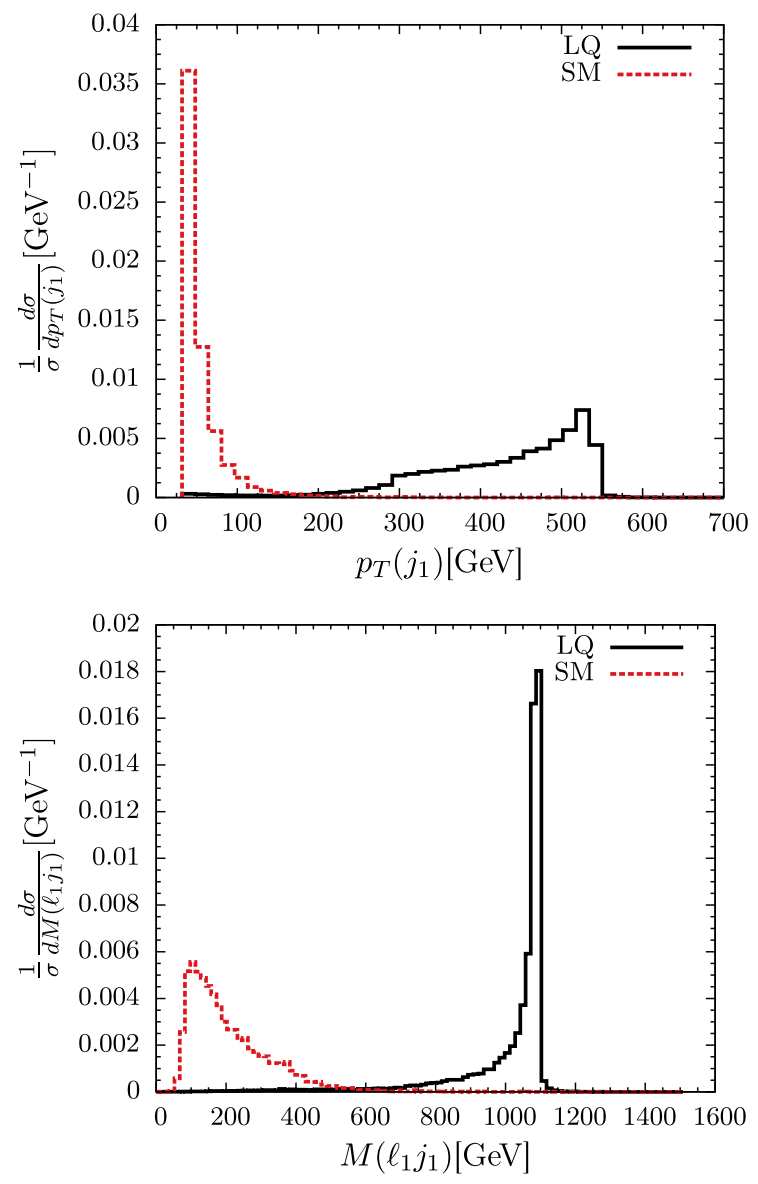

FIG. 7. Distribution of transverse momentum $p_{T}$ of the leading lepton, leading and subleading jet $p_{T}$ distribution, and invariant mass distribution of the leading lepton and leading jet for the final state $\ell^{-}+n-$ jets $(1 \leq n \leq 2)$. 

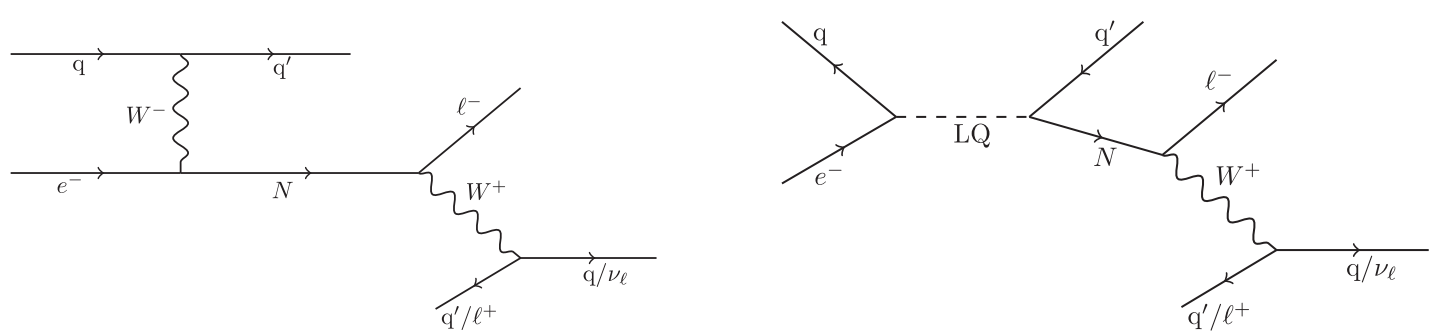

FIG. 8. Feynman diagram for various final states from $e^{-} p \rightarrow j N$. For this case, a $t$-channel LQ mediated diagram can also contribute.
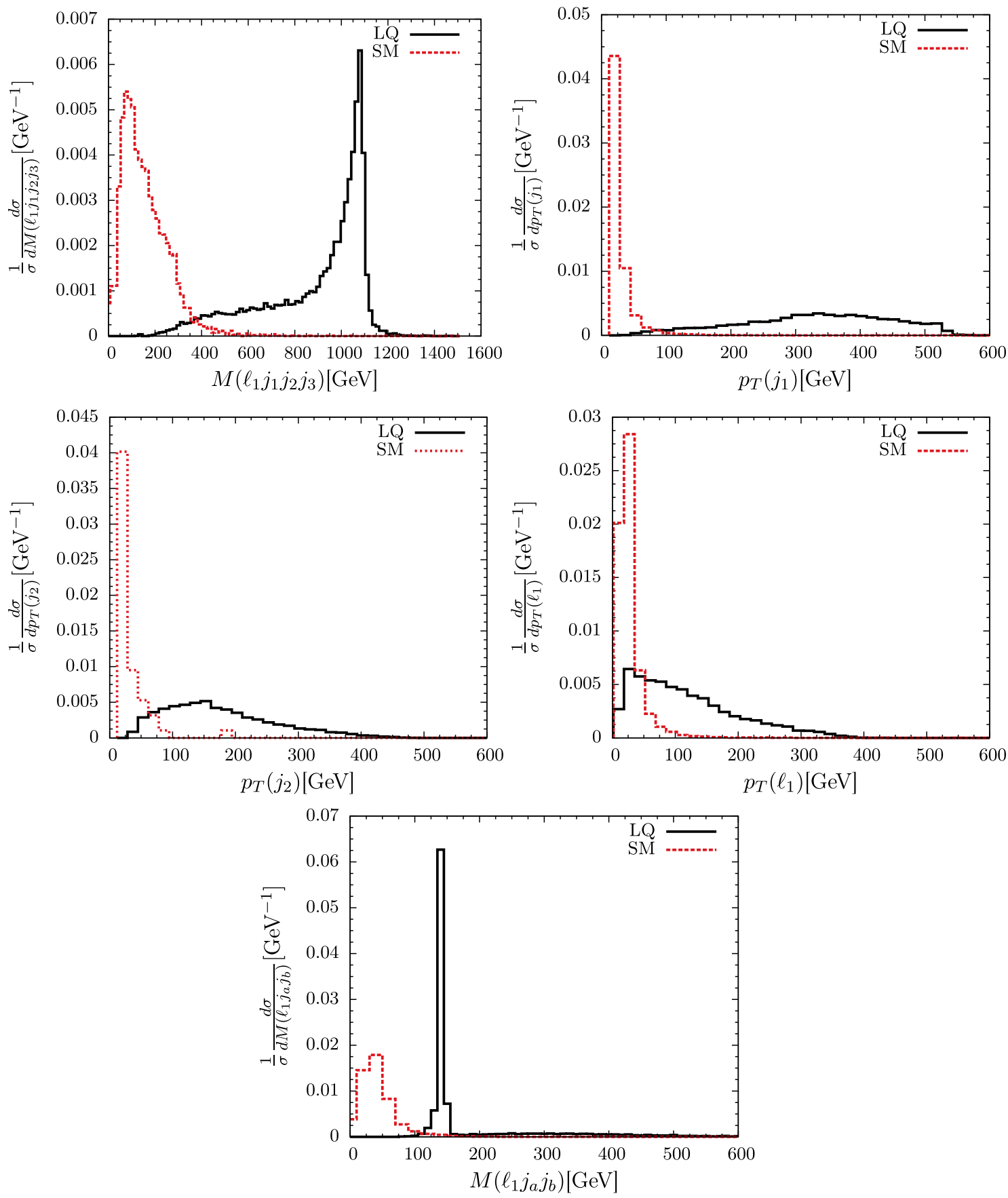

FIG. 9. Invariant mass distribution of a LQ $p_{T}$ distribution of leading, subleading jet, and of the leading lepton. We also show the invariant mass distribution of $N$ for the final state $\ell^{-}+n-$ jets $(n \geq 3)$. 
leading jet or lepton, and LQ invariant mass cut will reduce the SM background drastically. In Table. II, we have shown how we can reduce the SM background to zero using $p_{T}$ cut $-p_{T}^{\ell^{-}}(>400 \mathrm{GeV})$ on the leading lepton and invariant mass cut- $\left|M_{\mathrm{LQ}}-M_{\ell_{1} j_{1}}\right| \leq 100 \mathrm{GeV}$ simultaneously after using the basic sets of cuts mentioned in the previous section.

\section{B. Signal II}

If the coupling $Z_{i j}$ is nonzero, the LQ can also decay to a RH neutrino and a jet, as shown in the right panel of Fig. 8. The considered final state, can also arise from the $t$-channel $W$ exchange diagram as shown in left panel of Fig. 8. For active-sterile mixing $V \sim 10^{-2}-10^{-3}$, the contribution from the LQ, however, dominates. For example, with the $\mathrm{BP} 2$, the $\mathrm{CC}$ production cross section is $\approx 12.7 \mathrm{fb}$, while the production cross section from LQ decay is $\approx 240 \mathrm{fb}$. The subsequent decays of the RH neutrino, followed by hadronic and leptonic decays of gauge bosons gives rise to a number of partonic states that we list below.

(1) $\ell^{-}+n-$ jets $\left(n=3\right.$ ) (For hadronic decays of $W^{+}$)

(2) $\ell^{-}+\ell^{+}+n-\operatorname{jets}(n=1)+\mathbb{E}_{T}$ (For leptonic decays of $W^{+}$)

$$
\text { 1. } \ell^{-}+n-\operatorname{jets}(n \geq 3)
$$

For the case of the hadronic decays of the charged gauge boson, we demand a charged lepton and at least three jets as the final state. The invariant mass of the three jets and the charged lepton must be equal to that of the mass of the LQ. Hence, a cut on the invariant mass distribution allows the separation of the signal from the backgound. For the background we generate $e^{-} p \rightarrow l^{-}+$ $n-$ jets up to $n=3$. The distribution is given in the top left panel of Fig. 9. Additionally, the leading jet that is directly generated from LQ decay has a very high transverse momentum (see Fig. 9). Therefore, a large cut on the transverse momentum of the leading jet reduces the background. As can be seen from Table III, the large $p_{T}$ cut on the leading jet itself reduces the background by $\mathcal{O}\left(10^{4}\right)$. Further reduction in background is achieved though a cut on the invariant mass distribution of the heavy neutrino. Stringent cuts, such as the cuts on the invariant mass of the LQ and the heavy neutrino make the background negligibly small.

$$
\text { 2. } \ell^{-} \boldsymbol{\ell}^{+}+n-\operatorname{jets}(n \geq 1)+\mathbb{E}_{T}
$$

For the scenario, when a charged gauge boson produced in the decay of the RH neutrino decays leptonically, the signal will have 2 opposite sign charged leptons, jets (one or more), and missing energy. The dominant SM background comes from the processes like $e^{-} p \rightarrow \ell^{-} \ell^{+} j \nu_{\ell}$ and $e^{-} p \rightarrow \ell^{-} \ell^{+} j j \nu_{\ell}$. The reduction of the background in this case is achieved by applying proper cuts on the missing energy, transverse momentum of the leading jet, and effective mass $M_{\mathrm{EFF}}$. The $M_{\mathrm{EFF}}$ variable is defined as

$$
M_{\mathrm{EFF}}=\sum_{i} p_{T_{i}}^{j}+\sum_{i} p_{T_{i}}^{\ell}+\mathbb{E}_{T},
$$

where $p_{T_{i}}^{j}, p_{T_{i}}^{\ell}$ are the transverse momentum of the jet and lepton, and $\mathscr{E}_{T}$ is the missing transverse energy. We expect a hard distribution for $M_{\mathrm{EFF}}$, since the $p_{T}$ of the lepton and jets coming mostly from resonantly produced LQs (as the $t$-channel contribution is small) is significantly large. The peak of the $M_{\mathrm{EFF}}$ shifts towards higher values with increasing LQ mass. We have shown in Fig. 10 the missing energy $\mathscr{E}_{T}, M_{\mathrm{EFF}}$, leading jet, and leading lepton $p_{T}$ distributions. The effect of different cuts on the signal and background cross sections are given in Table IV.

\section{Signal III}

For the LQ mass more than $M_{N}+M_{t}$, it can further decay to $\bar{t} N_{3}$, which enables a final state with large lepton or large jet multiplicity, see Fig. 11. The large lepton multiplicity is promising due to the suppressed SM background. For the $\bar{t} N_{3}$ production channel, considering subsequent decays of $N_{3}$ and $\bar{t}$, where $N_{3}$ is assumed to decay to $\tau^{ \pm} W^{\mp}$, the following final states at the parton level are possible:

(1) $\bar{b} \ell^{-} \tau^{-} \ell^{+}+\mathbb{E}_{T}$ (for leptonic decays of both the $W$ bosons, $W^{-} \rightarrow \ell^{-} \nu, W^{+} \rightarrow \ell^{+} \nu$ ),

(2) $\bar{b} \ell^{-} \tau^{-}+n-$ jets $(n=2)+\mathscr{E}_{T}$ (for the $W$ boson decays, $W^{-} \rightarrow \ell^{-} \nu$ and $\left.W^{+} \rightarrow j j\right)$,

(3) $\bar{b} \tau^{-} \ell^{+}+n-$ jets $(n=2)+\mathscr{E}_{T}$ (for the $W$ boson decays, $W^{-} \rightarrow j j$ and $W^{+} \rightarrow \ell^{+} \nu$ ),

(4) $\bar{b} \tau^{-}+n-\operatorname{jets}(n=4)$ (for the $W$ boson decays, $W^{-} \rightarrow j j$ and $\left.W^{+} \rightarrow j j\right)$.

We do not consider the last final states in our study because of very small cross section and very large SM background due to large jet multiplicity.

TABLE III. Signal and background cross sections for the final state $\ell^{-}+n-$ jets $(n \geq 3)$ with cuts. BP2 has been used for this final state.

\begin{tabular}{lccc}
\hline \hline Cuts & Final states & Signal (fb) & Background (fb) \\
\hline No cuts & $\ell^{-}+n-$ jets $(n \geq 3)$ & 24.8 & $2.99 \times 10^{6}$ \\
Basic cuts & $\ell^{-}+n-$ jets $(n \geq 3)$ & 7.65 & $2.9 \times 10^{3}$ \\
Leading Jet $p_{T}$ cut & $+p_{T}^{j_{1}}(>200 \mathrm{GeV})$ & 6.56 & 180 \\
LQ invaraint mass cut & $+\left|M_{\mathrm{LQ}}-M_{\ell_{1} j_{1} j_{2} j_{3}}\right| \leq 100 \mathrm{GeV}$ & 3.65 & 60 \\
$N$ invaraint mass cut & $+\left|M_{N_{1}}-M_{\ell_{1} j_{a} j_{b}}\right| \leq 30 \mathrm{GeV}$ & 3.08 & 0 \\
\hline \hline
\end{tabular}



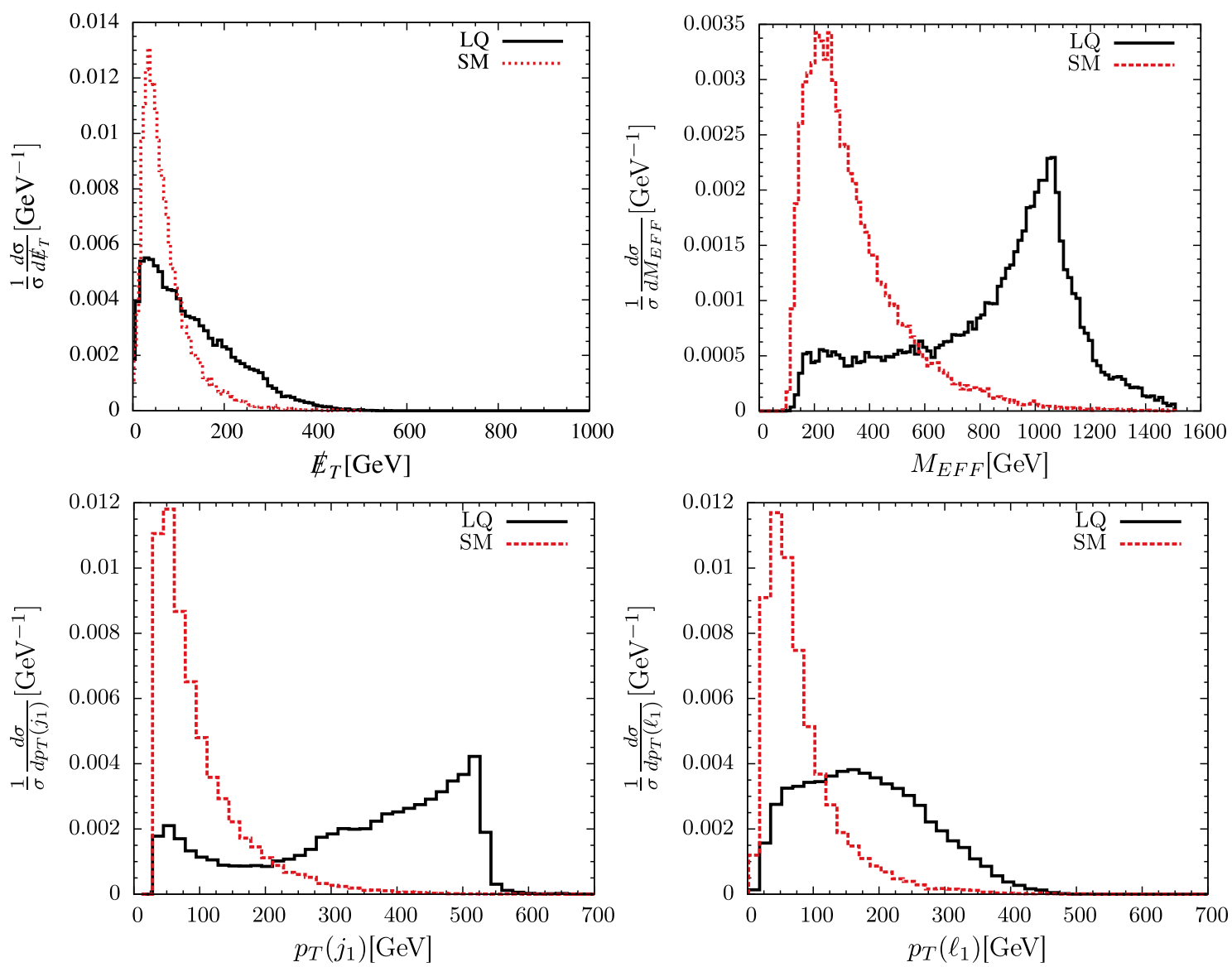

FIG. 10. Missing transverse energy distribution, $M_{\mathrm{EFF}}$ distribution, $p_{T}$ distribution of the leading jet, and leading lepton for the final state $\ell^{-} \ell^{+}+n-$ jets $(n \geq 1)+\mathbb{E}_{T}$.

$$
\text { 1. } \bar{b} \boldsymbol{\ell}^{-} \tau^{-} \boldsymbol{\ell}^{+}+\boldsymbol{E}_{T}
$$

For the final states involving $\tau$ and $b$, tagging can reduce the SM background significantly. We consider the $p_{T}$ for the $b$ and $\tau$ jets, as $p_{T}>40 \mathrm{GeV}$. In this work, we adopt a minimalistic approach and consider a flat $75 \%$ efficiency for $b$ tagging and 60\% efficiency for $\tau$ tagging. Similar to the previous case, the $M_{\mathrm{EFF}}$ distribution is hard due to the large missing energy and large transverse momenta of final state particles. For this signal, the most dominant SM backgound comes from the process $\bar{t} \ell^{-} W^{+}, \bar{t} Z \nu$ and $\bar{t} h \nu$ with $\bar{t} \rightarrow \bar{b} \tau^{-} \nu, W^{+} \rightarrow \ell^{+} \nu, Z \rightarrow \ell^{+} \ell^{-}$, and $h \rightarrow \ell^{+} \ell^{-}$.
After applying the basic cuts only, the SM background drops significantly. In addition, we use missing energy $\mathbb{E}_{T}$, leading jet $p_{T}$, and $M_{\mathrm{EFF}}$ distribution to further reduce the SM background.

$$
\text { 2. } \bar{b} \boldsymbol{e}^{-} \tau^{-}+n-\operatorname{jets}(n \geq 2)+\mathbb{E}_{T}
$$

For this case, due to large jet multiplicity, the SM background is greater than the previous signal and the background mainly comes from the process $\bar{t} \ell^{-} W^{+}$. However, using missing energy $\mathbb{E}_{T}$, cuts on leading jet $p_{T}$

TABLE IV. Signal and background cross section after various cuts for the final state $\ell^{-} \ell^{+}+n-$ jets $(n \geq 1)+\mathbb{E}_{T}$. BP2 has been used for this final state.

\begin{tabular}{lccc}
\hline \hline Cuts & Final states & Signal $(\mathrm{fb})$ & Background (fb) \\
\hline No cuts & $\ell^{-} \ell^{+}+n-$ jets $(n \geq 1)+\mathbb{E}_{T}$ & 11.2 & $5.22 \times 10^{2}$ \\
Basic cuts & $\ell^{-} \ell^{+}+n-\operatorname{jets}(n \geq 1)+\mathbb{E}_{T}$ & 7.84 & 258 \\
Missing energy cut & $\ell^{-} \ell^{+}+n-$ jets $(n \geq 1)+\mathbb{E}_{T}(>100 \mathrm{GeV})$ & 4.26 & 57.5 \\
Leading Jet $p_{T}$ cut & $+p_{T}^{j_{1}}(>300 \mathrm{GeV})$ & 3.24 & 3.73 \\
$M_{\mathrm{EFF}}$ cuts & $+M_{\mathrm{EFF}}(>500 \mathrm{GeV})$ & 2.88 & 2.54 \\
\hline \hline
\end{tabular}


and $M_{\mathrm{EFF}}$ distribution, the SM background can be reduced significantly.

$$
\text { 3. } \bar{b} \ell^{+} \tau^{-}+n-\operatorname{jets}(n \geq 2)+\mathbb{E}_{T}
$$

For this final state, the SM background is actually negligibly small at the $e^{-} p$ collider for the beam energies considered. The result is shown in Table VII.

\section{SIGNAL STRENGTH FOR HIGHER LQ MASS}

Bounds on LQs parameter space are expected to improve in the future with increasing luminosity at the LHC. Hence, we repeat our study for higher LQ masses. Though for higher LQ mass we can allow for large Yukawa coupling, we use the same coupling as for the 1.1 TeV LQ mass to compare our result for different LQ masses. Using the same set of cuts for each final state as we did for LQ mass 1.1 TeV, we have calculated the cross section for LQ mass of $1.2,1.3, \ldots$, up to $1.7 \mathrm{TeV}$. The partonic cross sections and the effect of different cuts are shown in Tables VIII and IX.

\section{A. Zero background case}

First, we consider only final states for which the SM background is zero or can be reduced to zero using an invariant mass cut of LQ and RH neutrinos. The SM background is practically zero for final state $\bar{b} \ell^{+} \tau^{-}+n-$ jets $(t n \geq 2)+\mathbb{E}_{T}$. For the final state $\ell^{-}+n-\operatorname{jets}(1 \leq n \leq 2)$, using $p_{T}^{\ell}$ cut and LQ invariant mass cut (for corresponding LQ mass), the SM background can be reduced to zero. Similarly, for the final state, $\ell^{-}+n-$ jets $(n \geq 3)$, selection cut on $p_{T}^{j}$, along with on LQ invariant mass, and the invariant mass of the RH neutrino can make the SM background negligibly small. The results are given in Table VIII. We have also shown the cross section as a function of LQ masses in Fig. 12. As can be seen, the cross section for the $\ell^{-}+n-$ jets $(1 \leq n \leq 2)$ channel is the largest and varies $10^{2}-0.42 \mathrm{fb}$ for a wide range of LQ mass. With $100 \mathrm{fb}^{-1}$ luminosity, this predicts $10^{4}$ number of events at $\mathrm{LHeC}$. The other channel with jet multiplicity

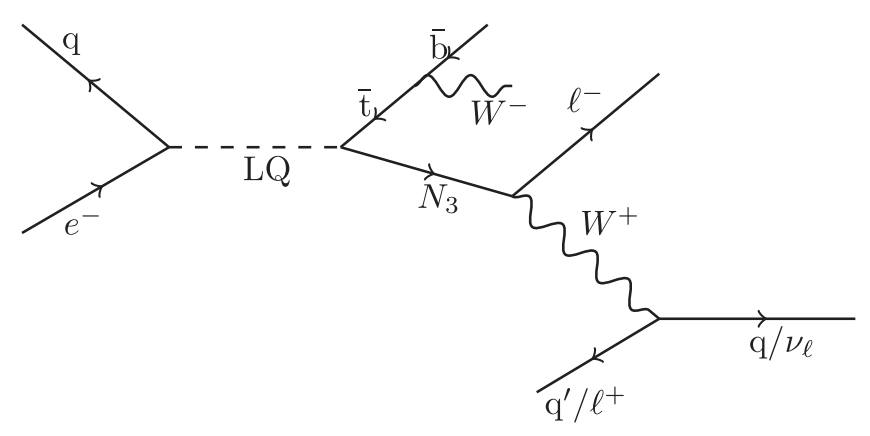

FIG. 11. Feynman diagram for various final states from $e^{-} p \rightarrow \bar{t} N_{3}$. For this case also, the $t$-channel mediated diagram with gauge boson and LQ will contribute.

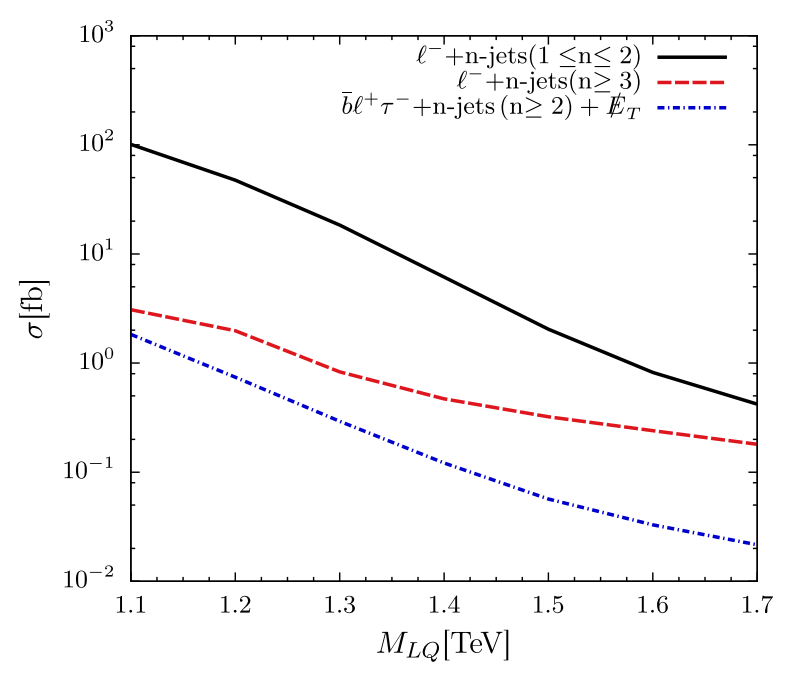

FIG. 12. Signal cross section as a function of LQ mass for different final states.

$(n \geq 3)$ also offers a large cross section, and a large number of events $\mathcal{O}\left(10^{2}\right)$.

\section{B. Nonzero background case}

For the final states, $\ell^{-}+n-$ jets $(1 \leq n \leq 2)$ and $\ell^{-}+n-$ jets $(n \geq 3)$, the SM background is nonzero if we do not use the invariant mass of the LQ and RH neutrino. Since the LQ and RH neutrino masses are unknown, we do not implement the mass cut, rather in this section we show the cross sections with a very generic set of cuts. Assuming LQ mass to be more than $1 \mathrm{TeV}$, all the other cuts that we considered can be easily applied. For the above two final states we applied cuts only on $p_{T}^{\ell}$ and $p_{T}^{j}$. For final states, $\ell^{-} \ell^{+}+n-$ jets $(n \geq 1)+\mathscr{E}_{T}$, $\bar{b} \ell^{-} \tau^{-} \ell^{+}+\mathbb{E}_{T}$ and $\bar{b} \ell^{-} \tau^{-}+n-$ jets $(n \geq 2)+\mathbb{E}_{T}$ we used the same cuts as in Tables IV, V, and VI, respectively. We show the signal cross section and statistical significance with integrated luminosity of $100 \mathrm{fb}^{-1}$ in Fig. 13. We also show the required luminosity to achieve $3 \sigma$ and $5 \sigma$ statistical significance in Fig. 14. The statistical significance has been calculated using the following expression:

$$
S_{\mathrm{sig}}=\frac{S}{\sqrt{S+B}}
$$

where, $S$ and $B$ denote the number of signal and background events, respectively.

\section{Results}

We discuss the discovery prospect of LQs in the mass range 1.1-1.7 $\mathrm{TeV}$ at $\mathrm{LHeC}$. We have shown the significance for different final states with integrated luminosity 100 and $1000 \mathrm{fb}^{-1}$ for each LQ mass in the range $1.1-1.7 \mathrm{TeV}$ in Table. $\mathrm{X}$. The channel $\ell^{-}+n-$ jets $(1 \leq n \leq 2)$ is the most promising. For the final states 
TABLE V. Signal and background cross section after various cuts for the final state $\bar{b} \ell^{-} \tau^{-} \ell^{+}+\mathbb{E}_{T}$. BP3 has been used for this final state.

\begin{tabular}{lccc}
\hline \hline Cuts & Final states & Signal $(\mathrm{fb})$ & Background $(\mathrm{fb})$ \\
\hline No cuts & $\bar{b} \ell^{-} \tau^{-} \ell^{+}+\mathscr{E}_{T}$ & 1.57 & 0.323 \\
Basic cuts & $\bar{b} \ell^{-} \tau^{-} \ell^{+}+\not \mathscr{E}_{T}$ & 0.83 & $7.51 \times 10^{-3}$ \\
Missing energy cut & $\bar{b} \ell^{-} \tau^{-} \ell^{+}+\mathbb{E}_{T}(>100 \mathrm{GeV})$ & 0.502 & $4.46 \times 10^{-3}$ \\
Leading Jet $p_{T}$ cut & $+p_{T}^{j_{1}}(>100 \mathrm{GeV})$ & 0.476 & $2.16 \times 10^{-3}$ \\
$M_{\mathrm{EFF}}$ cuts, $b$ and $\tau$ tagging & $\left.+M_{\mathrm{EFF}}>500 \mathrm{GeV}\right)$ & 0.21 & $7.7 \times 10^{-4}$ \\
\hline \hline
\end{tabular}

TABLE VI. Signal and background cross section after various cuts for the final state $\bar{b} \ell^{-} \tau^{-}+n-$ jets $(n \geq 2)+\not E_{T}$. BP3 has been used for this final state.

\begin{tabular}{lccc}
\hline \hline Cuts & Final states & Signal $(\mathrm{fb})$ & Background $(\mathrm{fb})$ \\
\hline No cuts & $\bar{b} \ell^{-} \tau^{-}+n-$ jets $(n \geq 2)+\mathbb{E}_{T}$ & 3.54 & 0.729 \\
Basic cuts & $\bar{b} \ell^{-} \tau^{-}+n-$ jets $(n \geq 2)+\mathbb{E}_{T}$ & 1.457 & $2.18 \times 10^{-2}$ \\
Missing energy cut & $\bar{b} \ell^{-} \tau^{-}+n-$ jets $(n \geq 2)+\mathbb{E}_{T}(>100 \mathrm{GeV})$ & 1.277 & $1.344 \times 10^{-2}$ \\
Leading Jet $p_{T}$ cut & $+p_{T}^{j_{1}}(>100 \mathrm{GeV})$ & 1.226 & $7.65 \times 10^{-3}$ \\
$M_{\text {EFF }}$ cuts, $b$ and $\tau$ tagging & $+M_{E F F}(>500 \mathrm{GeV})$ & 0.522 & $2.4 \times 10^{-3}$ \\
\hline \hline
\end{tabular}
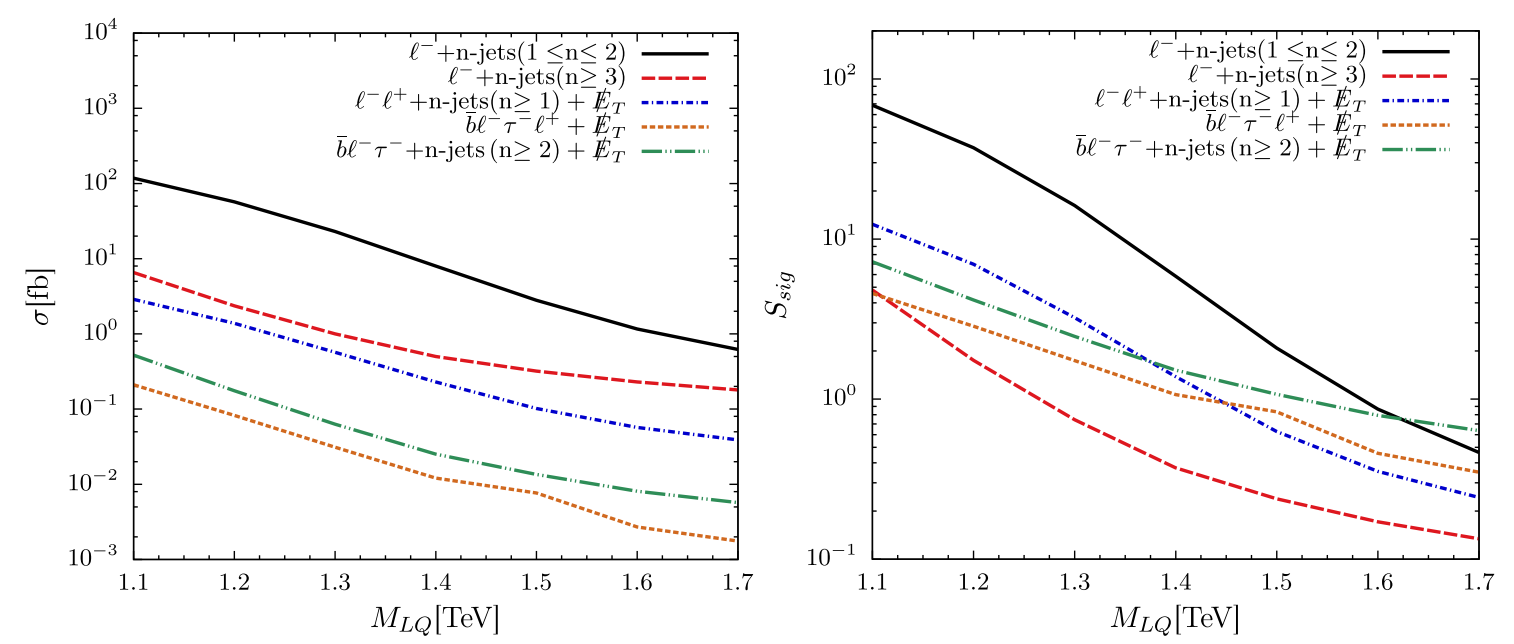

FIG. 13. Left panel: the signal cross section as a function of LQ mass for different final states. Right panel: the significance with $100 \mathrm{fb}^{-1}$ luminosity.
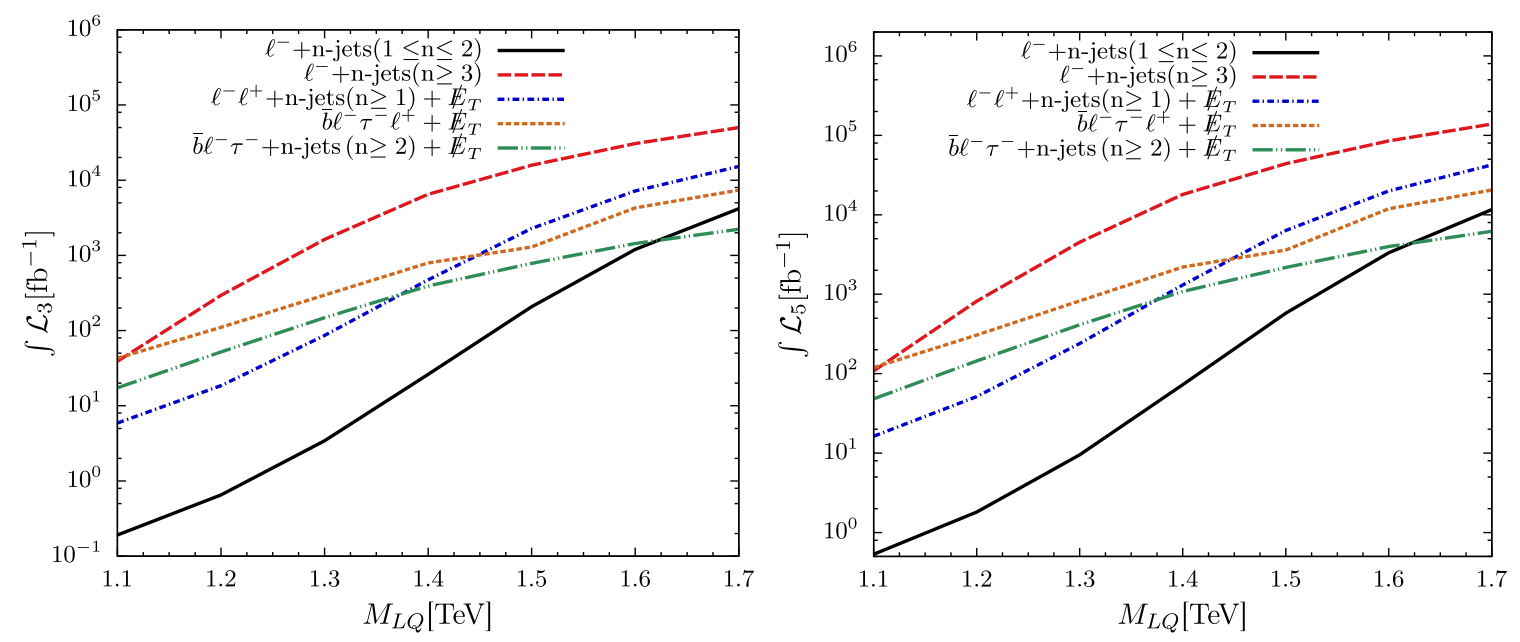

FIG. 14. The required luminosity to achieve $3 \sigma$ (left panel) and $5 \sigma$ (right panel) for different final states. 
TABLE VII. Signal and background cross section after various cuts for the final state $\bar{b} \ell^{+} \tau^{-}+n-$ jets $(n \geq 2)+\mathbb{E}_{T}$. BP3 has been used for this final state.

\begin{tabular}{lccc}
\hline \hline Cuts & Final states & Signal $(\mathrm{fb})$ & Background (fb) \\
\hline No cuts & $\bar{b} \ell^{+} \tau^{-}+n-\operatorname{jets}(n \geq 2)+\mathbb{E}_{T}$ & 4.07 & 0 \\
Basic cuts, b and $\tau$ tagging & $\bar{b} \ell^{+} \tau^{-}+n-\operatorname{jets}(n \geq 2)+\mathbb{E}_{T}$ & 1.83 & 0 \\
\hline \hline
\end{tabular}

TABLE VIII. Cross sections (in fb) after all the cuts as a function of LQ mass.

\begin{tabular}{lccccccc}
\hline \hline Final states & $1.1 \mathrm{TeV}$ & $1.2 \mathrm{TeV}$ & $1.3 \mathrm{TeV}$ & $1.4 \mathrm{TeV}$ & $1.5 \mathrm{TeV}$ & $1.6 \mathrm{TeV}$ & $1.7 \mathrm{TeV}$ \\
\hline$\ell^{-}+n-\operatorname{jets}(1 \leq n \leq 2)$ & 101 & 47.53 & 18.37 & 6.148 & 2.04 & 0.82 & 0.42 \\
$\ell^{-}+n-\operatorname{jets}(n \geq 3)$ & 3.08 & 1.98 & 0.83 & 0.47 & 0.322 & 0.24 & 0.18 \\
$\bar{b} \ell^{+} \tau^{-}+n-\operatorname{jets}(n \geq 2)+\boldsymbol{E}_{T}$ & 1.83 & 0.74 & 0.29 & 0.12 & 0.06 & 0.03 & 0.02 \\
\hline \hline
\end{tabular}

TABLE IX. Cross sections in fb, after all the cuts (except invariant LQs and right-handed neutrinos mass cut) as a function of LQ mass. Backgrounds are same as for $1.1 \mathrm{TeV}$ as the beam energies are same.

\begin{tabular}{lccccccc}
\hline \hline Final states & $1.1 \mathrm{TeV}$ & $1.2 \mathrm{TeV}$ & $1.3 \mathrm{TeV}$ & $1.4 \mathrm{TeV}$ & $1.5 \mathrm{TeV}$ & $1.6 \mathrm{TeV}$ & $1.7 \mathrm{TeV}$ \\
\hline$\ell^{-}+n-$ jets $(1 \leq n \leq 2)$ & 118 & 57 & 23 & 8 & 2.8 & 1.16 & 0.62 \\
$\ell^{-}+n-$ jets $(n \geq 3)$ & 6.56 & 2.36 & 1 & 0.5 & 0.32 & 0.23 & 0.18 \\
$\ell^{-} \ell^{+}+n-\operatorname{jets}(n \geq 1)+\mathbb{E}_{T}$ & 2.88 & 1.38 & 0.57 & 0.23 & 0.102 & 0.057 & 0.039 \\
$\bar{b} \ell^{-} \tau^{-} \ell^{+}+\mathbb{E}_{T}$ & 0.21 & 0.08 & 0.03 & 0.012 & 0.007 & 0.003 & 0.0018 \\
$\bar{b} \ell^{-} \tau^{-}+n-\operatorname{jets}(n \geq 2)+\mathbb{E}_{T}$ & 0.522 & 0.175 & 0.063 & 0.025 & 0.013 & 0.008 & 0.006 \\
\hline \hline
\end{tabular}

TABLE X. Significance for different final states with integrated luminosity $\mathcal{L}=100 \mathrm{fb}^{-1}$ (1st column corresponding to each LQ mass) and $\mathcal{L}=1000 \mathrm{fb}^{-1}$ (2nd column corresponding to each LQ mass), respectively, for the LQ mass range [1.1-1.7] TeV.

\begin{tabular}{|c|c|c|c|c|c|c|c|c|c|c|c|c|c|c|}
\hline \multirow{2}{*}{$\begin{array}{l}\text { Final states } \\
\ell^{-}+n-\text { jets }(1 \leq n \leq 2)\end{array}$} & \multicolumn{2}{|c|}{$1.1 \mathrm{TeV}$} & \multicolumn{2}{|c|}{$1.2 \mathrm{TeV}$} & \multicolumn{2}{|c|}{$1.3 \mathrm{TeV}$} & \multicolumn{2}{|c|}{$1.4 \mathrm{TeV}$} & \multicolumn{2}{|c|}{$1.5 \mathrm{TeV}$} & \multicolumn{2}{|c|}{$1.6 \mathrm{TeV}$} & \multicolumn{2}{|c|}{$1.7 \mathrm{TeV}$} \\
\hline & 68 & 217 & 37 & 117 & 16 & 51 & 6 & 19 & 2 & 7 & 0.87 & 2.74 & 0.46 & 1.46 \\
\hline$\ell^{-}+n-\operatorname{jets}(n \geq 3)$ & 4.8 & 15 & 1.74 & 5.5 & 0.74 & 2.35 & 0.37 & 1.17 & 0.24 & 0.75 & 0.23 & 0.54 & 0.13 & 0.42 \\
\hline$\ell^{-} \ell^{+}+n-\operatorname{jets}(n \geq 1)+E_{T}$ & 12 & 39 & 7 & 22 & 3.2 & 10 & 1.4 & 4.4 & 0.62 & 1.98 & 0.35 & 1.12 & 0.24 & 0.76 \\
\hline $\bar{b} \ell^{-} \tau^{-} \ell^{+}+\mathbb{E}_{T}$ & 4.5 & 14.4 & 2.8 & 8.9 & 1.7 & 5.4 & 1.06 & 3.3 & 0.8 & 2.5 & 0.5 & 1.5 & 0.35 & 1.12 \\
\hline $\bar{b} \ell^{-} \tau^{-}+n-\operatorname{jets}(n \geq 2)+\mathbb{E}_{T}$ & 7.2 & 22 & 4.15 & 13 & 2.46 & 7.8 & 1.5 & 4.77 & 1.04 & 3.3 & 0.78 & 2.48 & 0.65 & 2.1 \\
\hline
\end{tabular}

$\ell^{-}+n-$ jets $(1 \leq n \leq 2)$, even with integrated luminosity $2 \mathrm{fb}^{-1}$, the statistical significance is $9.69 \sigma$ for the LQ of mass $1.1 \mathrm{TeV}$.

For final states $\ell^{-}+n-\operatorname{jets}(n \geq 3)$ and $\ell^{-} \ell^{+}+n-$ jets $(n \geq 1)+\mathbb{E}_{T}$, statistical significance drastically decreases with higher LQ masses. Similarly for the final states $\bar{b} \ell^{-} \tau^{-} \ell^{+}+\mathbb{E}_{T}$ and $\bar{b} \ell^{-} \tau^{-}+n-\operatorname{jets}(n \geq 2)+\mathbb{E}_{T}$, in spite of the small SM background, as the signal cross section is itself small for higher LQ masses, it is difficult to observe these final states for higher LQ masses.

\section{CONCLUSIONS}

In this work, we study the discovery prospect of the $\tilde{R}_{2}$ class of the LQ model at the LHeC. The model contains two LQs with $Q=\frac{2}{3}$ and $Q=-\frac{1}{3}$. The LQ with $Q=\frac{2}{3}$ can be copiously produced at $\mathrm{LHeC}$ due to its interaction with the electron and down-type quark. We study the production and its decay to different final states, including a lepton and a jet, a jet and a RH neutrino, and a RH neutrino and a top quark. The typical production cross sections for $e^{-} p \rightarrow$ $l j, j N_{1}, \bar{t} N_{3}$ are 221,242 , and $222 \mathrm{fb}$ for $M_{\mathrm{LQ}}=1.1 \mathrm{TeV}$, $M_{N_{1,3}}=150 \mathrm{GeV}$, and the couplings $Y_{11}=0.3, Z_{33}=1$. The produced $\mathrm{RH}$ neutrino further decays and gives a plethora of model signatures. For the RH neutrinos, we adopt a model independent framework, and a large activesterile mixing to ensure its decay within the detector. For the LQs, the higher production cross section as well as the lower backgrounds at the $\mathrm{LHeC}$ result in a much higher statistical significance for a few of the signals studied.

We have analyzed a number of final states, including $\ell^{-}+n-$ jets $\quad(1 \leq n \leq 2), \quad \ell^{ \pm}+n-$ jets $(n \geq 3), \quad \ell^{ \pm} \ell^{\mp}+n-$ jets $(n \geq 1)+\mathbb{E}_{T}, \bar{b} \ell^{-} \tau^{-} \ell^{+}+\mathbb{E}_{T}, \bar{b} \ell^{-} \tau^{-}+n-$ jets $(n \geq 2)+$ $\mathbb{E}_{T}, \bar{b} \ell^{+} \tau^{-}+n-$ jets $(n \geq 2)+\mathbb{E}_{T}$. Among these, the model 
signature $\ell^{-}+n-$ jets $(1 \leq n \leq 2)$ arises due to the direct decay of LQ to a lepton and a jet. All the other final states arise due to the decay of the LQ to a RH neutrino and a light jet, or to a RH neutrino and top quark, with successive decays of the RH neutrino, and a $t$ quark into SM states.

We find that, among all the above mentioned final states, $\ell^{-}+n-$ jets $(1 \leq n \leq 2)$ has the highest discovery prospect even after giving a generic set of cuts. A LQ of mass up to $1.4 \mathrm{TeV}$ in this channel can be discovered at more than $5 \sigma$ C.L. with $100 \mathrm{fb}^{-1}$ of data. The LQs will also result in the enhancement of the $\mathrm{RH}$ neutrino production in association with a light jet, or with a top quark. If at $\mathrm{LHeC}$ the electron beam is polarized, the right-handed neutrino-light jet production cross section can substantially increase [46,47]. We find that among all the final states $\ell^{-}+n-$ jets $(n \geq 3)$, and $\bar{b} \ell^{+} \tau^{-}+n-$ jets $(n \geq 2)+\mathbb{E}_{T}$ are the most optimal, after implementing the selection cuts judiciously. With $100 \mathrm{fb}^{-1}$ integrated luminosity, for LQ mass $1.1 \mathrm{TeV}$, the expected number of events for the final sates $\ell^{-}+n-$ jets $(n \geq 3)$, and $\bar{b} \ell^{+} \tau^{-}+n-$ jets $(n \geq 2)+\mathbb{E}_{T}$ are $10^{4}$ and 180 , respectively.

\section{ACKNOWLEDGMENTS}

M. M. would like to acknowledge the DST-INSPIRE research Grant No. IFA14-PH-99.
[1] J. C. Pati and A. Salam, Phys. Rev. D 10, 275 (1974).

[2] W. Buchmuller, R. Ruckl, and D. Wyler, Phys. Lett. B 191, 442 (1987); 448, 320 (1999).

[3] W. Buchmuller and D. Wyler, Phys. Lett. B 177, 377 (1986).

[4] J. C. Pati and A. Salam, Phys. Rev. D 8, 1240 (1973).

[5] H. Georgi and S. L. Glashow, Phys. Rev. Lett. 32, 438 (1974).

[6] H. Georgi, AIP Conf. Proc. 23, 575 (1975).

[7] H. Fritzsch and P. Minkowski, Ann. Phys. (N.Y.) 93, 193 (1975).

[8] R. N. Mohapatra and B. Sakita, Phys. Rev. D 21, 1062 (1980).

[9] F. Wilczek and A. Zee, Phys. Rev. D 25, 553 (1982).

[10] A. Melfo, A. Ramirez, and G. Senjanovic, Phys. Rev. D 82, 075014 (2010).

[11] K. S. Babu and R. N. Mohapatra, Phys. Rev. Lett. 70, 2845 (1993).

[12] B. Bajc, G. Senjanovic, and F. Vissani, Phys. Rev. Lett. 90, 051802 (2003).

[13] A. S. Joshipura and K. M. Patel, Phys. Rev. D 83, 095002 (2011).

[14] P. S. B. Dev and R. N. Mohapatra, Phys. Rev. D 81, 013001 (2010).

[15] B. Bajc, G. Senjanovic, and F. Vissani, Phys. Rev. D 70, 093002 (2004).

[16] S. Dimopoulos and L. Susskind, Nucl. Phys. B155, 237 (1979).

[17] S. Dimopoulos, Nucl. Phys. B168, 69 (1980).

[18] E. Farhi and L. Susskind, Phys. Rep. 74, 277 (1981).

[19] H. Georgi and S. L. Glashow, Phys. Rev. Lett. 47, 1511 (1981).

[20] I. Doršner, S. Fajfer, A. Greljo, J. F. Kamenik, and N. Košnik, Phys. Rep. 641, 1 (2016).

[21] J. M. Arnold, B. Fornal, and M. B. Wise, Phys. Rev. D 87, 075004 (2013).

[22] P. Minkowski, Phys. Lett. 67B, 421 (1977).

[23] R. N. Mohapatra and G. Senjanovic, Phys. Rev. Lett. 44, 912 (1980).

[24] M. Gell-Mann, P. Ramond, and R. Slansky, Conf. Proc. C790927, 315 (1979).
[25] T. Yanagida, Conf. Proc. C7902131, 95 (1979).

[26] J. Schechter and J. W. F. Valle, Phys. Rev. D 25, 774 (1982).

[27] J. Schechter and J. W. F. Valle, Phys. Rev. D 22, 2227 (1980).

[28] S. Weinberg, Phys. Rev. Lett. 43, 1566 (1979).

[29] S. Weinberg, Phys. Rev. D 22, 1694 (1980).

[30] M. Magg and C. Wetterich, Phys. Lett. 94B, 61 (1980).

[31] T. P. Cheng and L.-F. Li, Phys. Rev. D 22, 2860 (1980).

[32] R. Foot, H. Lew, X. G. He, and G. C. Joshi, Z. Phys. C 44, 441 (1989).

[33] R. N. Mohapatra, Phys. Rev. Lett. 56, 561 (1986).

[34] S. Nandi and U. Sarkar, Phys. Rev. Lett. 56, 564 (1986).

[35] R. N. Mohapatra and J. W. F. Valle, Phys. Rev. D 34, 1642 (1986).

[36] M. Mitra, G. Senjanovic, and F. Vissani, Nucl. Phys. B856, 26 (2012).

[37] M. Malinsky, J. C. Romao, and J. W. F. Valle, Phys. Rev. Lett. 95, 161801 (2005).

[38] M. Hirsch, S. Morisi, and J. W. F. Valle, Phys. Lett. B 679, 454 (2009).

[39] J. Barry, W. Rodejohann, and H. Zhang, J. High Energy Phys. 07 (2011) 091.

[40] J. Heeck and H. Zhang, J. High Energy Phys. 05 (2013) 164.

[41] S. K. Kang and C. S. Kim, Phys. Lett. B 646, 248 (2007).

[42] S. K. Majee, M. K. Parida, and A. Raychaudhuri, Phys. Lett. B 668, 299 (2008).

[43] M. K. Parida and A. Raychaudhuri, Phys. Rev. D 82, 093017 (2010).

[44] D. Das, K. Ghosh, M. Mitra, and S. Mondal, Phys. Rev. D 97, 015024 (2018).

[45] U. K. Dey, D. Kar, M. Mitra, M. Spannowsky, and A. C. Vincent, Phys. Rev. D 98, 035014 (2018).

[46] S. Mondal and S. K. Rai, Phys. Rev. D 94, 033008 (2016).

[47] S. Mondal and S. K. Rai, Phys. Rev. D 93, 011702 (2016).

[48] M. Lindner, F. S. Queiroz, W. Rodejohann, and C. E. Yaguna, J. High Energy Phys. 06 (2016) 140.

[49] S. Antusch, E. Cazzato, and O. Fischer, Int. J. Mod. Phys. A 32, 1750078 (2017). 
[50] J. L. Abelleira Fernandez et al. (LHeC Study Group), J. Phys. G 39, 075001 (2012).

[51] J. L. Abelleira Fernandez et al. (LHeC Study Group), arXiv:1211.5102.

[52] G. Aad et al. (ATLAS Collaboration), Eur. Phys. J. C 72, 2056 (2012).

[53] G. Aad et al. (ATLAS Collaboration), J. High Energy Phys. 07 (2015) 162.

[54] S. Chatrchyan et al. (CMS Collaboration), Phys. Lett. B 717, 109 (2012).

[55] V. Khachatryan et al. (CMS Collaboration), J. High Energy Phys. 04 (2016) 169.

[56] A. M. Sirunyan et al. (CMS Collaboration), Phys. Rev. Lett. 120, 221801 (2018).

[57] A. Atre, T. Han, S. Pascoli, and B. Zhang, J. High Energy Phys. 05 (2009) 030.

[58] A. M. Sirunyan et al. (CMS Collaboration), arXiv:1806 .10905.

[59] A. Abada, C. Biggio, F. Bonnet, M. B. Gavela, and T. Hambye, J. High Energy Phys. 12 (2007) 061.

[60] A. Caputo, P. Hernandez, J. Lopez-Pavon, and J. Salvado, J. High Energy Phys. 06 (2017) 112.

[61] A. Das, Adv. High Energy Phys. 2018, 9785318 (2018).

[62] A. Das and N. Okada, Phys. Rev. D 88, 113001 (2013).

[63] A. Das and N. Okada, Phys. Lett. B 774, 32 (2017).

[64] J. Chakrabortty, S. Goswami, and A. Raychaudhuri, Phys. Lett. B 698, 265 (2011).

[65] S. Banerjee, P. S. B. Dev, A. Ibarra, T. Mandal, and M. Mitra, Phys. Rev. D 92, 075002 (2015).

[66] H.-J. Behrend et al., Phys. Lett. B 178, 452 (1986).

[67] G. Abbiendi et al. (OPAL Collaboration), Eur. Phys. J. C 13, 15 (2000).

[68] P. Abreu et al. (DELPHI Collaboration), Phys. Lett. B 446, 62 (1999).

[69] G. Abbiendi et al. (OPAL Collaboration), Phys. Lett. B 526, 233 (2002).

[70] G. Abbiendi et al. (OPAL Collaboration), Eur. Phys. J. C 31, 281 (2003).

[71] I. Abt. et al. (H1 Collaboration), Nucl. Phys. B396, 3 (1993).

[72] C. Adloff et al. (H1 Collaboration), Eur. Phys. J. C 11, 447 (1999); 14, 553 (2000).

[73] S. Chekanov et al. (ZEUS Collaboration), Phys. Rev. D 68, 052004 (2003).

[74] F. D. Aaron et al. (H1 Collaboration), Phys. Lett. B 701, 20 (2011).

[75] F. D. Aaron et al. (H1 Collaboration), Phys. Lett. B 704, 388 (2011).

[76] H. Abramowicz et al. (ZEUS Collaboration), Phys. Rev. D 86, 012005 (2012).

[77] S. Abachi, Phys. Rev. Lett. 72, 965 (1994).

[78] V. M. Abazov et al. (D0 Collaboration), Phys. Lett. B 668 , 357 (2008).
[79] V. M. Abazov et al. (D0 Collaboration), Phys. Lett. B 671, 224 (2009).

[80] V. M. Abazov et al. (D0 Collaboration), Phys. Lett. B 693, 95 (2010).

[81] V. M. Abazov et al. (D0 Collaboration), Phys. Rev. D 84, 071104 (2011).

[82] F. e. a. Abe (CDF Collaboration), Phys. Rev. D 48, R3939 (1993).

[83] D. Acosta et al. (CDF Collaboration), Phys. Rev. D 72, 051107 (2005).

[84] A. Abulencia et al. (CDF Collaboration), Phys. Rev. D 73, 051102 (2006).

[85] T. Aaltonen et al. (CDF Collaboration), Phys. Rev. D 77, 091105 (2008).

[86] V. Khachatryan et al. (CMS Collaboration), Phys. Rev. D 93, 032004 (2016).

[87] G. Aad et al. (ATLAS Collaboration), Eur. Phys. J. C 76, 5 (2016).

[88] A. M. Sirunyan et al. (CMS Collaboration), J. High Energy Phys. 07 (2017) 121.

[89] M. Aaboud et al. (ATLAS Collaboration), New J. Phys. 18, 093016 (2016).

[90] A. M. Sirunyan et al. (CMS Collaboration), Phys. Rev. D 98, 032005 (2018).

[91] A. M. Sirunyan et al. (CMS Collaboration), J. High Energy Phys. 07 (2018) 115.

[92] F. Romeo (ATLAS and CMS Collaborations), Proceedings of the 2017 International Workshop on Baryon and Lepton Number Violation: From the Cosmos to the LHC (BLV 2017) Cleveland, Ohio, USA, 2017 (Case Western Reserve University, Cleveland, 2017).

[93] I. Dorsner, S. Fajfer, and A. Greljo, J. High Energy Phys. 10 (2014) 154.

[94] I. Dorsner, J. Drobnak, S. Fajfer, J. F. Kamenik, and N. Kosnik, J. High Energy Phys. 11 (2011) 002.

[95] A. Alloul, N. D. Christensen, C. Degrande, C. Duhr, and B. Fuks, Comput. Phys. Commun. 185, 2250 (2014).

[96] J. Alwall, R. Frederix, S. Frixione, V. Hirschi, F. Maltoni, O. Mattelaer, H. S. Shao, T. Stelzer, P. Torrielli, and M. Zaro, J. High Energy Phys. 07 (2014) 079.

[97] T. Sjostrand, S. Mrenna, and P. Z. Skands, J. High Energy Phys. 05 (2006) 026.

[98] M. Cacciari, G. P. Salam, and G. Soyez, J. High Energy Phys. 04 (2008) 063.

[99] M. Cacciari, G. P. Salam, and G. Soyez, Eur. Phys. J. C 72, 1896 (2012).

Correction: The previously published Figure 4 contained a plotting error and has been replaced. 